Cahiers $d u$ MONDE RUSSE

\section{Cahiers du monde russe}

Russie - Empire russe - Union soviétique et États indépendants

\section{$56 / 1 \mid 2015$}

Fictions d'avenir : sciences et temps des socialismes est-européens

\title{
Les horloges suspendues du futur
}

Les mondes de la science-fiction en Bulgarie et en Roumanie socialistes

Time on hold: science fiction worlds in socialist Bulgaria and Romania

\section{Nadège Ragaru et Antonela Capelle-Pogăcean}

\section{(2) OpenEdition}

\section{Journals}

Édition électronique

URL : http://journals.openedition.org/monderusse/8167

DOI : $10.4000 /$ monderusse. 8167

ISSN : $1777-5388$

Éditeur

Éditions de l'EHESS

Édition imprimée

Date de publication : 1 janvier 2015

Pagination : $77-109$

ISBN : 978-2-7132-2475-1

ISSN : $1252-6576$

Référence électronique

Nadège Ragaru et Antonela Capelle-Pogăcean, « Les horloges suspendues du futur », Cahiers du monde russe [En ligne], 56/1 | 2015, mis en ligne le 13 juillet 2019, consulté le 10 octobre 2020. URL : http://journals.openedition.org/monderusse/8167 ; DOI : https://doi.org/10.4000/monderusse.8167

Ce document a été généré automatiquement le 10 octobre 2020

(c) École des hautes études en sciences sociales 


\title{
Les horloges suspendues du futur
}

\author{
Les mondes de la science-fiction en Bulgarie et en Roumanie socialistes
}

Time on hold: science fiction worlds in socialist Bulgaria and Romania

\author{
Nadège Ragaru et Antonela Capelle-Pogăcean
}

1 L'univers de la science-fiction ${ }^{1}$ ne manque pas d'attraits pour qui souhaite contribuer à une histoire culturelle du socialisme et de ses temporalités. Comme genre, que ce soit dans ses déclinaisons littéraire, cinématographique ou graphique, la science-fiction est tout entière portée par une pensée du temps : elle investit le futur et le passé en tant que parabole du présent et/ou cherche à donner sens aux destinées humaines au croisement entre science et métaphysique. Or le socialisme fut aussi, par excellence, un projet de maîtrise du temps tendu vers un horizon téléologique. Ce projet, nourri des Lumières $\mathrm{du} \mathrm{XVIII}^{\mathrm{e}}$ siècle et des ambitions de connaissance de la nature à des fins d'émancipation $\mathrm{du} \mathrm{XIX}^{\mathrm{e}}$ siècle, se voulait ordonné par des lois scientifiques. Quoi de plus évident, dès lors, que de chercher à placer en regard ces deux ingénieries du temps et de l'homme?

Retenir ce prisme présente une seconde vertu heuristique : sous le socialisme, la science-fiction a occupé une place dont Sonija Fritsche a justement montré dans le cas est-allemand qu'elle était à la fois périphérique (au regard des censeurs et des mondes de l'écriture) et centrale (en termes de popularité auprès des lectorats, mais aussi dans l'œuvre de popularisation de la science) ${ }^{2}$. Ce double statut en fait un site privilégié d'observation des quotidiens socialistes, a fortiori si l'on se souvient que les mondes de la science-fiction (clubs, cénacles et festivals) s'imposèrent dans les années 1980 comme des univers de socialisation fréquentés par des groupes sociaux et générationnels y voyant une ouverture sur le monde. À bien des égards, le genre constitue ici une sphère d'intérêt ambiguë, dont il semble parfois délicat de déterminer si elle fut encouragée, tolérée et/ou réprimée par les pouvoirs publics est-européens. Que ces derniers l'aient envisagée (ou non) comme une alternative à la futurologie occidentale ${ }^{3}$ n'interdit nullement à ses auteurs, amateurs et lecteurs de l'avoir investie comme un espace de liberté et/ou de contestation. Enfin, la définition de l'objet retenue - à savoir non seulement les œuvres dans leur matérialité, leurs conditions de production, de 
circulation et de réception, mais aussi les univers des clubs - présente l'avantage de traverser l'horizon spatial des découpages conventionnels de la guerre froide.

Envisagée depuis le ciel, la terre voit en effet s'estomper les partages géopolitiques du temps court. Elle s'offre en même temps à des imaginations nourries par des histoires plurielles. À l'Est comme à l'Ouest, quoique parfois selon des cheminements distincts, l'institutionnalisation du genre science-fictionnel est en effet contemporaine de l'entrée dans une « ère cosmique » mondiale avec le vol de Sputnik (1957), puis, dans les années 1970, d'une interrogation souvent partagée sur l'anticipation des défis globaux (la maîtrise de la croissance, l'épuisement des ressources, la menace d'une catastrophe écologique...). En d'autres termes, les planètes de la science-fiction autorisent une écriture historienne de la " petite différence » invitant à cerner le singulier au sein de strates plus larges.

Telle est également l'une des raisons qui a informé le choix d'un rapprochement entre les trajectoires bulgare et roumaine. Dans ces deux États à bien des égards inégalement insérés au sein du " bloc de l'Est », s'observe en effet au tournant des années 1950-1960 - date à laquelle nous avons choisi de faire débuter notre récit - une reconnaissance institutionnelle de la science-fiction qui coïncide avec une période d'enthousiasme cosmique et de libéralisation politique. Cette symbiose est toutefois médiatisée par des inscriptions internationales contrastées : là où en Bulgarie la proximité avec l'Union soviétique et avec une langue russe maîtrisée par la majorité des lecteurs constitue le vecteur d'une ouverture sur les univers du futur soviétiques comme occidentaux, en Roumanie c'est davantage par le truchement de contacts ouest-européens, la France et l'Italie en particulier, que la découverte s'opère en une période de désoviétisation. Les deux pays n'en voient pas moins se dessiner des horizons européens qui échappent partiellement à l'opposition entre États-Unis et Union soviétique. Dans un État comme dans l'autre, la production d'un avenir socialiste se conjugue en outre, de manière croissante à compter des années 1970, avec un ressourcement dans un passé national venant relocaliser le temps. Ces choix communs voilent cependant des évolutions en ciseaux : partiellement synchronisées pendant la décennie de libéralisation des années 1960, les horloges politiques bulgare et roumaine se désolidarisent dans les décennies suivantes ; la désillusion est plus précoce, plus profonde aussi en Roumanie que dans une Bulgarie où les univers science-fictionnels continueront jusqu'à la fin des années 1980 à débattre des options de remise en marche d'un temps socialiste autre.

5 Fondé sur le dépouillement de sources archivistiques (Comités à la culture, archives de clubs de science-fiction), des entretiens (avec des auteurs, traducteurs, éditeurs et amateurs), des mémoires, des écrits (fanzines, revues spécialisées, ouvrages et anthologies) et des sources secondaires, l'examen croisé des terrains bulgare et roumain offrira deux apports à la littérature sur l'histoire culturelle des socialismes tardifs. Le premier concerne l'étude des scansions temporelles du socialisme, singulièrement de ses modulations internes et de ses déclinaisons générationnelles. Là où, dans l'élan des années 1960, la science-fiction sert de véhicule à une projection plutôt confiante dans l'avenir, au niveau individuel comme collectif, au tournant des années 1970-1980 les textes localement produits et/ou importés accueillent une inquiétude et un repli dans un hors temps alternativement situé en un passé lointain et un futur inaccessible. En demeurer à ce constat serait toutefois erroné. Car, par-delà la logique d'institutionnalisation d'un genre et donc de la création des cadres de sa reproduction, une des raisons pour lesquelles la science-fiction comme littérature et 
comme réseau de sociabilité a connu une large popularité dans les années 1970-1980 réside en ce qu'elle a fourni un espace de débat en des sociétés que l'on avait aspiré à produire homogènes et qui se découvraient diversifiées. Les univers d'amateurs de science-fiction ont ainsi vu évoluer côte à côte des tenants d'une lecture rationaliste du monde, des esprits sensibles au paranormal et à l'ésotérisme, des curieux désireux de valoriser une pensée rock punk ou tout simplement non-standard. Du ferment de leurs frustrations et de leurs enthousiasmes est née une créativité qui interdit de réduire la science-fiction à une fuite dans la dystopie.

6 À un second niveau, l'expédition en terre de science-fiction offre l'opportunité de penser à nouveaux frais les ancrages spatiaux des socialismes est-européens. En l'occurrence, les cas bulgare et roumain donnent à voir une concomitance entre le développement d'une pensée globale du temps et le déploiement d'un travail de nationalisation, entendu ici à la fois comme ressourcement identitaire et vernacularisation d'un genre. La valorisation de la nation des années 1970 rend ainsi également possible une ouverture placée au service de la reconnaissance internationale par patrimonialisation des œuvres dont la circulation hors frontières est soutenue. Sans être la seule variable explicative, ce désir de reconnaissance conduit à doter les

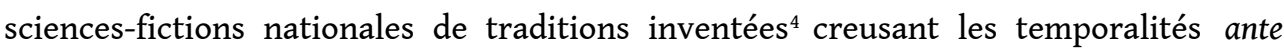
communistes. La production internationale de "nationalités littéraires» SF s'accompagne ainsi d'un enracinement du genre ${ }^{5}$ au moment même où les peuples bulgare et roumain sont projetés dans des ethnogenèses de plus en plus profondes.

7 Afin de déployer cette argumentation, nous procéderons en deux temps, qui recouvrent grossièrement des découpages chronologiques, l'ère de l'enthousiasme cosmique (de la fin des années 1950 jusqu'au début des années 1970) et le temps dystopique d'un socialisme tardif (jusqu'à la fin de la décennie 1980). Chaque moment reliera les thématisations du temps dans les écrits de science-fiction, dans les mondes sociaux des clubs et les productions politiques du temps. Ces voyages donneront à voir des disjonctions de plus en plus sensibles entre les divers registres de présence du futur passé et leurs expériences générationnellement situées.

\section{Les années 1960-1970 : la science-fiction du rêve cosmique, un genre en quête de légitimité}

8 Le lancement de Sputnik, le premier vol habité dans l'espace, les premiers pas hésitants d'un humain sur la Lune rythment une ère de rivalité entre puissances qui est également une période d'enthousiasme pour le cosmos. Cette fascination soutient tant en Bulgarie qu'en Roumanie la reconnaissance de la science-fiction littéraire. Fenêtre sur les représentations sociales du temps, ce nouveau genre donne également un aperçu des quotidiens socialistes à travers les lieux de socialisation que furent les clubs de SF.

\section{La cristallisation d'une écriture du futur au temps de la conquête spatiale}

Dans des déclinaisons inspirées du fantastique et du rêve ${ }^{6}$, de l'épouvante ${ }^{7}$, de l'anticipation et de l'exploration scientifique ${ }^{8}$, une écriture de l'exaltation et de l'inquiétude scientifiques avait connu une certaine faveur en Bulgarie et en Roumanie à 
partir du début du $\mathrm{Xx}^{\mathrm{e}}$ siècle. En Roumanie, le journaliste et astronome amateur Victor Anestin publiait en 1899 un roman En l'an 4000 ou un Voyage sur Vénus, alors que son ami Henri Stahl envoyait en 1914 Un Roumain sur la Lune. En Bulgarie c'est essentiellement après la Première Guerre mondiale qu'une poignée de lettrés polyglottes s'étaient essayés à l'invention d'un monde fantastique imprégné de diabolisme, à l'instar du journaliste et prosateur Svetoslav Minkov dont la plume associait à l'imagination de voyages lunaires (L'horloge, 1922) une lecture parodique et sombre de l'automatisation des sociétés (La dame aux yeux rayons X, 1934).

Rétrospectivement, les histoires des SF nationales allaient annexer à leur entreprise généalogique des auteurs dont les productions avaient couvert une large palette de genres, comme Felix Aderca, poète symboliste, traducteur en roumain de la pièce R.U.R. de Karel Čapek (1926) ou le conteur et folkloriste bulgare Elin Pelin dont le Jan Bibijan et la lune (1932-1933) allait habiter les imaginaires de plusieurs générations d'enfants. Là où Aderca arpentait la voie de la dystopie avec ses Villes Submergées (1935), le symboliste Georgi Iliev rêvait dans O-Kors (1930) une planète glacée. Rien de comparable, donc, dans ces deux pays à l'éclosion de la naučna fantastika (littéralement : fantastique scientifique) observée en Russie dès la fin du xix siècle qui allait connaître de foisonnantes ramifications jusqu'à ce que Stalin impose un recadrage idéologique au profit de la seule « fantasy du jour prochain » à la fin des années $1930^{\circ}$.

11 Après la Seconde Guerre mondiale, c'est en ce contexte soviétique hostile à la science-fiction que la Bulgarie et la Roumanie amorcent une soviétisation politique et culturelle. Jusqu'au milieu des années 1950, sur fond de guerre froide, les échappées spatio-temporelles sont stigmatisées en tant que divertissement futile associé à l'Occident; les quelques écrits publiés sont des traductions depuis le russe, qui dépeignent les miracles des avancées technologiques du socialisme. Le futur proche guide les récits, l'avenir lointain demeure interdit. Sous la plume des auteurs soviétiques ou de leurs avatars nationaux, la figure de l'espion capitaliste (américain), les yeux rivés sur les découvertes soviétiques, fait par ailleurs frémir les âmes adolescentes, principaux destinataires du genre.

Bien que les maisons d'édition (et la large diffusion des revues soviétiques en Bulgarie) aient fait découvrir à leurs lecteurs les premiers écrits d'Ivan Efremov dès l'immédiat après-guerre, l'entrée dans l'ère cosmique assure à la science-fiction une visibilité éditoriale inédite ${ }^{10}$. Assurément, l'enthousiasme stellaire n'y revêt pas la même ampleur que dans une Union soviétique où la conquête spatiale a été investie comme indice du triomphe du socialisme et support de fierté patriotique ${ }^{11}$. En Bulgarie comme en Roumanie, grandit cependant une " génération Sputnik ${ }^{12}$ dont les imaginaires et les goûts seront durablement modelés par la référence à l'espace. De cet éblouissement plus marqué au sud du Danube, l'un des futurs animateurs du club de science-fiction " Ivan Efremov », Todor Jalămov, alors élève au lycée de langue russe de Sofia, s'est fait l'écho : « Tous les journaux avaient annoncé l'heure du survol de Sofia. Une humanité déferla dans les rues pour fêter la petite étoile au-dessus de nous. [...] À l'entrée du jardin du roi Boris, d'innombrables amateurs d'étoiles pointaient du doigt la furtive lumière nouvelle $»^{13}$. Le premier vol habité en 1961 suscite une liesse collective ${ }^{14}$. En l'espace de quelques années, les murs des écoles se parent de peintures cosmiques, les villes (bulgares surtout) de monuments et de statuaires, les poches adolescentes d'images, timbres et autres objets fétiches échangés avec jubilation, ainsi des 
photographies de Jurij Gagarin acclamé devant le mausolée Georgi Dimitrov à Sofia le 24 mai $1961^{15}$.

13 La déstalinisation (la " désoviétisation » aussi en Roumanie) et l'amorce de la détente favorisent l'institutionnalisation de la science-fiction au croisement entre popularisation de la science, romans d'aventures, policiers et d'espionnage, écrits fantastiques et utopies technologiques et sociales ${ }^{16}$. Trois vecteurs de diffusion se dessinent alors qui concernent les collections SF des éditeurs souvent labellisés " jeunesse », les revues de vulgarisation scientifique et, plus timidement, des maisons d'édition généralistes venant glisser dans leurs catalogues des figures jouissant d'une internationale renommée. Une politique des honneurs (l'organisation de concours du " meilleur récit » par des revues de popularisation de la science) contribue également à l'établissement du genre.

14 Au tournant des années 1970, la gamme des éditeurs s'élargit : la science-fiction pénètre le milieu des éditions généralistes et obtient la consécration d'éditions spécialisées, plus visibles toutefois en Bulgarie qu'en Roumanie. Dans ce pays, en 1971, les Éditions Albatros (ex-Éditions de la Jeunesse) se dotent de la collection « Fantastic club", les éditions pour enfants lancent une collection "Jules Verne", tandis que " Universul ", maison consacrée à la « littérature universelle », accueille une collection intermittente du roman scientifico-fantastique. En Bulgarie, au-delà de la création par l'éditeur « La patrie » d'une collection de " Naučna fantastika » en 1977, une étape essentielle est franchie en 1979 avec la fondation à Varna d'une édition entièrement dédiée à la science-fiction, Narodna biblioteka Galaktika. Référence adulée aux tirages épuisés sitôt parus et aux volumes amoureusement collectionnés ${ }^{17}$, Galaktika publiera quelque 110 titres, dont les tirages oscillent entre 40000 et $100000^{18}$. Le format de poche et les couvertures aux couleurs du rêve associées à de sensuelles (extra)humanités séduisent les lecteurs au moins autant que la modestie des coûts et la qualité de choix éditoriaux internationalisés ${ }^{19}$. Grâce à leur concision, les récits de science-fiction irriguent aussi peu à peu le monde de revues généralistes, y compris celles appartenant à l'Union des écrivains ${ }^{20}$. L'économie des écritures favorise par ailleurs le développement d'anthologies servant à légitimer le genre dans une perspective nationale (construction d'une généalogie et publication de jeunes auteurs) et internationale (ces anthologies participant d'un ordonnancement par espace linguistique des mondes $\mathrm{SF}$ ).

15 Le croisement des paysages bulgare et roumain dessine ainsi un monde dans lequel la science-fiction, écriture originellement labellisée pour la jeunesse, diffusée par des supports largement semblables et popularisée à travers des concours, s'ouvre progressivement à un lectorat adulte, certaines œuvres réalisant des tirages remarquables $^{21}$. Cette restitution des étapes de l'institutionnalisation de la science-fiction invite en outre à dépasser les lectures du genre en termes de politisation de la production culturelle offertes dans maints récits autochtones. Une première approche retrace l'histoire de la SF à travers le prisme de la répression et du répertoire contestataire (les pouvoirs publics auraient été par définition réticents envers les pensées alternatives et toute projection vers un futur prétendant se substituer aux analyses marxistes) ; un autre courant fait a contrario de la science-fiction un genre qui aurait été envisagé par les autorités communistes comme un relais idéologique au service de la compétition globale Est-Ouest à travers l'affirmation d'un autre futur. Le lancement d'éditions, la publication d'œuvres semblent refléter davantage l'oscillation 
des pouvoirs publics entre le désir de diffuser le goût des sciences et d'encourager une inventivité scientifique y compris à travers le volet créatif de la science-fiction, et la crainte que cette dernière, genre léger d'évasion, ne contrevienne aux exigences de la formation de la jeunesse. Fruit des mobilisations d'une partie des protagonistes du champ, l'autorisation de collections est aussi la conséquence de la popularité d'une littérature investie, tant par ses producteurs que ses lecteurs, comme un espace de pensée-interrogation. Ces intelligences plurielles du genre ressortiront plus avant si nous envisageons maintenant les modes de thématisation des Ailleurs spatio-temporels offerts dans les œuvres science-fictionnelles.

\section{Chronos en terre de naučna fantastika à l'heure des libéralisations politiques}

En matière de thématiques et de lectures du temps, une première période s'étend du tournant des années 1950 jusqu'au milieu des années 1960 ; la seconde se clôt vers la fin de la décennie 1970. Au commencement étaient la maîtrise des destinées scientifiques et les acquis sociétaux de la connaissance. Née en contexte de déstalinisation et d'esquisse de détente, la première génération d'œuvres de science-fiction vernaculaire transpose volontiers les rivalités avec l'Occident dans un futur imaginaire, mais elle en envisage généralement la résolution sur le mode de la fraternité : il s'agit d'une écriture du contact, de la rencontre, voire de la coopération. Néanmoins, le franchissement des frontières géopolitiques a pour arrière-plan l'instauration d'une distinction entre une science du futur impérialiste et dominatrice, incarnée par les États-Unis, et une science vertueuse, visant l'accomplissement de l'homme et le progrès de l'humanité. Emblématique de cette époque est L'homme atomique du jeune littérateur bulgare Ljuben Dilov, édité en 1958 dans la collection "Aventures et science-fiction » de l'éditeur Jeunesse populaire. L'ouvrage projette en un lointain avenir (communiste) de coopération scientifique Est-Ouest, un Américain, Jimmy Cook [sic !] qui, après avoir participé à la chasse aux sorcières des années 1950, s'est retrouvé pris dans les glaces de l'Antarctique au cours d'expérimentations sur la bombe à hydrogène ; il revient à la vie pour découvrir l'absurdité de son ancienne idéologie. Le déplacement temporel a ici vocation cathartique dans le temps même où il souligne la centralité des Américains comme significant other ${ }^{22}$.

17 À l'orée des années 1960, le futur science-fictionnel se présente souvent comme un futur possible, proche ou accompli, auquel lecteurs et auteur sont reliés par un élan projectif, y compris lorsque sont creusés les sillons du passé. Cette littérature de l'espoir est émaillée d'injonctions aux vertus morales du socialisme. Un amour en l'an 41042, roman de Crişan Făgeraşu (alias Sergiu Fărcăşanu), dépeint ainsi l'homme nouveau, perfection de "statue antique" sortie des brumes d'un futur déjà advenu. Situé en l'an 50 000, le narrateur plonge en 41042 pour y retrouver son héros Ols, sauveur de l'astronef L'Arche de Noé et d'ancêtres égarés dans l'espace depuis l'an $9000^{23}$. L'on retrouve également cette image du corps parfait venu du futur dans la nouvelle de Vladimir Colin, Giovanna et l'ange, où l'amour entre la poétesse Giovanna et l'immortel Vittorio " aux yeux gris », scellé sur les ruines de Pompéi, porte la promesse d'une humanité pacifiée dans la jeunesse éternelle : « ... nous serons les nouveaux Prométhée qui apporteront l'étincelle de l'immortalité sur la Terre !». Le temps est à « une humanité nouvelle, qui ne menace rien et continue tout $»^{24}$. 

temps d'un socialisme en voie d'accomplissement. L'encouragement apporté par les pouvoirs communistes aux écritures amateurs incite des professionnels de la science et des techniques à prendre la plume. La libéralisation conduit également des écrivains communistes, " hommes nouveaux» nés dans les années 1920-1930 dont l'enthousiasme pour le nouvel ordre politique s'est assagi, à aborder les espaces lunaires. Ils se sont fait connaître antérieurement par des écrits pour enfants, des romans (d'aventure ou non), des épopées historiques, des écrits poético-folkloriques, ou même du théâtre (Pavel Vežinov, Haim Oliver ou, différemment, Jordan Radičkov, en Bulgarie, Vladimir Colin, Adrian Rogoz, Victor Kernbach ou Ion Hobana en Roumanie). Plus rares sont les auteurs venus du monde d'hier, à l'instar du Bulgare Svetoslav Minkov à qui les autorités laissent le loisir de poursuivre ses activités littéraires d'avant-guerre à la condition d'occuper une position subalterne au sein du champ et de se contenter de son emploi de bibliothécaire à la Bibliothèque nationale de Sofia. Le statut de la science-fiction au cours de cette première période - monde d'entre-deux qui aspire à une reconnaissance - rend possible ce croisement des hommes.

La palette des tonalités change à compter du milieu des années 1960, alors que la certitude du progrès technologique se charge d'inquiétudes. La science-fiction se mue alors progressivement en site privilégié d'expression du doute métaphysique. Aux côtés d'écrits aventuriers visant un lectorat adolescent, amateur d'expéditions et de science merveilleuse (Petăr Bobev en Bulgarie, I.M. Stefan, alias Alexandru Sergiu Sragher en Roumanie) se développe une littérature de la parabole où le voyage dans l'espace, les fonds marins ou les confins du globe sert une réflexion sur les modèles sociaux et écologiques ${ }^{25}$. Dans une frange des écrits, l'ailleurs temporel devient un instrument d'anticipation et de prévention des risques associés à l'accélération des mutations scientifiques, à la complexification des sociétés et/ou à l'épuisement des ressources de la planète. L'Autel des dieux stochastiques d'Adrian Rogoz, primé à Trieste en 1972, appartient à la première catégorie. L'auteur place le crash du " tachymètre » à cerveau électronique traversant l'Alabama à $950 \mathrm{~km} / \mathrm{h}$ sous les auspices de John Steinbeck : "Après des années de luttes, nous découvrons que ce n'est pas nous qui conduisons le voyage, mais lui qui nous conduit». Dans son sillage, Homer Hidden constate avec tristesse que " c'est cela qui avait gâché sa vie : la continuelle, folle accélération " ${ }^{26}$. Une même tristesse suicidaire habite l'architecte de villes verticales invisibles d'Alambaï ou les arcanes de l'art (1967, Adrian Rogoz ${ }^{27}$ ). Objet d'une enquête de l'office de l'ordre mondial en tant qu'inventeur "d'une cabine de reconversion en ondes" dont les passagers sont transformés en mouches par suite d'une avarie, l'inventeur s'efface entouré d'un nuage de diptères. En Bulgarie, avec une douce mélancolie, Le lézard blanc de Pavel Vežinov (1977) dépeint, lui, les désillusions d'un père, scientifique ordinaire de l'espoir, dont le fils surdoué se révèle dépourvu de sensibilité humaine. Devenu précocement grand-père (son fils ayant accédé à la paternité à l'âge de 13 ans), il se penche affligé sur le berceau du nouveau-né : "quoique déçu par son fils, il avait continué à espérer secrètement qu'avec lui était née une race humaine nouvelle extra-joyeuse comme on l'appelait parfois. Ce but était vain, illusoire et dénué de sens [...] car le développement humain ne connaît qu'une seule voie, naturelle $\aleph^{28}$. De cette accélération volontariste des changements (socialistes ?) qui masque la perversion des êtres, Vežinov fait une métaphore de l'incompréhension intergénérationnelle. 
20 Sur le plan des intrigues et des mécanismes de l'Ailleurs retenus, les sciences-fictions bulgare et roumaine ne se différencient pas alors radicalement de leurs homologues occidentales. Certes, on trouve peu ici cette science-fiction postapocalyptique dont l'écriture avait été suscitée à l'Ouest par la bombe atomique et le souvenir de la Shoah. Mais la dissemblance se limite le plus souvent à un léger décalage temporel. Ainsi la science-fiction de conscience écologique, dont les premiers écrits avaient été inspirés à l'Ouest par les travaux du Club de Rome au début des années 1970, s'inscrit-elle dans les horizons imaginaires bulgare et roumain plutôt à la fin de la décennie. Les écritures fictionnelles locales sont également en prise sur les interrogations globales relatives à la maîtrise scientifique du futur, voire, en Roumanie, sur les théories de la convergence $^{29}$; certains auteurs sont même directement impliqués dans les espaces de débats où Est et Ouest discutent au tournant des années 1970 de la prospective et des « future(s) studies $»^{30}$. Diplomate, adjoint du ministre des Affaires étrangères et ministre de l'Éducation, mathématicien et philosophe des sciences, Mircea MaliŢa joue ainsi un rôle pivot ${ }^{31}$ dans l'organisation du Third World Future Research Congress dédié à " l'avenir commun de l'homme» à Bucarest en 1972 ${ }^{32}$. D'autres écrivains de SF, singulièrement en Bulgarie où l'insertion internationale dans les réseaux globaux de la futurologie reste plus modeste ${ }^{33}$, les découvrent à travers la traduction d'ouvrages soviétiques ou occidentaux, ainsi que les recensions locales d'écrits européens et américains $^{34}$. Le vocabulaire même atteste ce décalage : en Bulgarie comme en URSS, l'on inscrit ces réflexions dans le champ de la prognostika, qui se pose en alternative à la futurologie occidentale. À Sofia, point de Centre international de méthodologie des sciences sur le futur et le développement semblable à celui qui existera entre 1974 et 1977 en Roumanie ${ }^{35}$, les discussions sur les sciences du futur, le pronostic social et le social planning demeurent faiblement institutionnalisées dans le cadre de l'Académie des sciences ${ }^{36}$ ou de l'Institut de planification du Comité central.

21 Avec une fréquence croissante, par ailleurs, les auteurs de SF bulgares et roumains investissent les voyages spatio-temporels moins pour réfléchir à un avenir à encadrer que pour en faire un instrument de réflexion sur le présent, à travers une opération de mise en étrangeté cognitive dont Darko Suvin a suggéré qu'elle constituait l'un des traits distinctifs du genre ${ }^{37}$. Les mobilités servent alors à décrire les égarements de régimes politiques aisés à reconnaitre, au-delà de leur transposition dans l'espace. La contrainte politique pesant sur l'écriture en régime autoritaire et l'art de la lecture interstitielle développé par les publics -coutumiers de la transposition des intrigues dans l'histoire afin de dépeindre les travers de l'ordre établi - facilitent cette exploration des ressources critiques d'un futur. L'existence de précédents, ainsi des écrits des frères Strugackij qui n'hésitent pas à transplanter sur la planète Arkanar un monde semi-féodal persécutant ses intellectuels (Dalëkaja Raduga, 1964), confère à cette option un caractère d'évidence. Le mouvement qui porte l'écriture depuis la technologie vers les enjeux sociaux de la science accompagne par ailleurs un glissement des héros collectifs - numériquement plus fréquents dans la production de masse locale que dans les écrits occidentaux de la même époque - vers des individualités dotées d'une épaisseur psychologique.

22 Émerge ainsi une méditation sur l'homme et ses abîmes, un homme qui se révèle non entièrement programmable, ainsi dans la nouvelle de Ion Hobana « Des hommes et des étoiles » (1965) où un personnage essaie de saboter une expédition sur Mars non parce qu'il serait un ennemi externe ou interne, mais parce que, soudainement, la peur 
s'empare de lui ${ }^{38}$. " Le voyage d'Icare » de Ljuben Dilov, primé à l'Eurocon de 1976, explore de même l'âme de Zenon Balov, premier homme né à bord d'un astéroïde, qui s'initie aux mystères d'Icare sans parvenir à trouver sa place dans le cosmos et disparaît happé par un trou noir ${ }^{39}$. Conjointement, l'intérêt science-fictionnel pour le voyage spatial tend à évoluer. Là où précédemment, la narration prenait pour point de départ l'envol, le trajet lui-même et le contact entre sociétés, les écrits s'intéressent désormais davantage au retour, alors que des astronautes regagnant la terre après un séjour (multi)séculaire dans l'espace se découvrent inadaptés au monde qu'ils avaient quitté, victimes de « cosmo-névroses $»^{40}$.

$\mathrm{Au}$ cours de cette période, pourtant, ni la Bulgarie, ni la Roumanie ne connaissent la désillusion cosmique qui s'empare de l'URSS dans le sillage du décès mystérieux des héros d'un programme spatial onéreux, émaillé de secrets ${ }^{41}$. Pour deux raisons au moins : en ces pays, c'est à la fin des années 1970 que les (plus modestes) bénéfices symboliques du projet spatial se manifestent, les premiers astronautes bulgares et roumains s'envolant vers le cosmos en 1977 et en $1981^{42}$. En outre, l'élan des années de libéralisation, d'affirmation d'une voie nationale vers le socialisme et de relatif bien-être économique contribue à envelopper le rêve spatial d'un voile de sérénité terrestre. De cette sérénité, les clubs et cénacles, institutions sans équivalent dans les autres genres littéraires en termes de popularité, ouvrent un site d'observation remarquable, où se prolongent lectures et expériences du futur.

\section{Des univers de sociabilité : les clubs de science-fiction}

En Bulgarie comme en Roumanie, l'institutionnalisation de la science-fiction sous la forme de clubs et " cénacles» intervient au croisement de plusieurs priorités de politique publique qui concernent l'investissement des sciences et de la culture par la jeunesse, l'éclosion d'activités amateurs et le façonnage de personnalités " multilatéralement développées». Le paysage SF demeure fluctuant, souvent tributaire de l'engagement d'individualités charismatiques et du calendrier des vies étudiantes. Un réseau de clubs se développe dès la fin des années 1960 en Roumanie, au milieu des années 1970 en Bulgarie ${ }^{43}$, dont le nombre ne cessera d'augmenter pour atteindre respectivement une cinquantaine et environ quatre-vingts en $1989^{44}$.

À bien des égards, les trajectoires sont ici semblables : les clubs sont le plus souvent initiés par des étudiants et de jeunes ingénieurs, mathématiciens, physiciens passionnés qui quêtent légitimité, locaux et budgets auprès de responsables des Jeunesses communistes et des maisons de la culture en s'appuyant sur le patronage de figures institutionnelles de la $\mathrm{SF}^{45}$. Les leaders du mouvement amateur cultivent l'éclectisme de formations scientifiques aux ambitions de plume; la présence de militaires et de figures liées à l'Union soviétique par leurs origines familiales, formation ou métier distingue toutefois la Bulgarie de la Roumanie ${ }^{46}$. Les clubs sont hébergés dans des maisons de la jeunesse ou de la culture des syndicats, parfois aussi des universités, lycées et entreprises ${ }^{47}$. Leurs activités mêlent lectures et débats, projections filmiques et écoute musicale, conférences et expositions d'arts SF, ateliers d'écriture et édition. Au fil des années 1970, un maillage d'instances de rencontre se tisse, la première Romcon (officiellement appelée " Conférence nationale ») se tenant à Bucarest en 1972, son homologue Bulgarcon à Sofia six ans plus tard. Chaque année, des festivals, journées et camps d'été réunissent amateurs, scientifiques, écrivains, directeurs de collection, 
critiques et invités de marque étrangers. Les joies visuelles d'expositions et de films ${ }^{48}$ alternent avec des tables rondes (sur l'histoire du genre, l'avenir des sciences ou les OVNI) ; l'organisation de soirées musicales prolongées tard dans la nuit constitue souvent le second volet, non-officiel mais d'autant plus séduisant, du programme des rencontres. En un temps où la culture du divertissement amorce une entrée timide dans les jeunes existences bulgares et roumaines, cet univers des clubs fournit ainsi un espace de socialisation dont la valeur est accrue par le sentiment d'appartenir à un monde d'initiés.

Sites de réception des écrits, les clubs constituent par ailleurs des lieux de production, où de jeunes aspirants auteurs font circuler les premières versions de leurs textes. Certains éditent des fanzines ${ }^{49}$, contournant les obstacles à la publication posés par des " littéraires", défenseurs des contours " canoniques» du territoire alors que les ressources (notamment de papier) se font rares ${ }^{50}$. Si la parution aléatoire caractérise les fanzines bulgares et roumains situés dans un limes entre domaine institutionnel et univers informel, les titres bulgares jouissent parfois de tirages élevés ${ }^{51}$ sans toutefois acquérir la reconnaissance internationale de leurs homologues roumains. En 1972, Solaris reçoit ainsi le prix spécial du jury au premier Eurocon organisé à Trieste ; sa couverture est reproduite dans le programme de la convention. Repris sous forme de recueils, les récits d'Omicron obtiennent en 1980 le prix « Europe » de la "meilleure revue d'amateurs du continent » à la convention européenne de Stresa ; enfin Helion et Paradox seront récompensés lors des Eurocon de 1984 et 1987.

Trois contrastes "spatio-temporels» affectent cependant la chronologie de l'institutionnalisation des clubs, les ancrages symboliques du cosmos et, incidemment, les visions politisées du futur. En Roumanie, l'émergence des clubs est contemporaine d'une période d'ouverture internationale et de relative libéralisation politique ; en Bulgarie l'élan de cette dernière est, un temps, brisé par l'écrasement du Printemps de Prague. L'attitude des pouvoirs bulgares est empreinte d'ambivalence, oscillant entre le souci d'inciter la jeunesse à s'emparer des miracles de la science et une réticence devant des initiatives collectives menaçant d'échapper au contrôle politique. Il faut souvent s'assurer la médiation de figures " politiquement sûres " - à l'instar de Jurij Ilkov, officier en charge des questions politiques à la garnison de Pazardžik - pour que les autorités locales concèdent l'octroi d'une salle à l'aménagement sommaire. Outre le climat politique, la chronologie décalée reflète une structure différenciée des ressources politiques, culturelles et sociales dont disposent les animateurs du mouvement SF.

En Roumanie, l'institutionnalisation des cénacles, tout comme la production d'une science-fiction à même de gagner des lettres de noblesse, bénéficie du soutien de personnalités littéraires établies au sein de l'Union des écrivains et sur la scène internationale. Ion Hobana, directeur de collection SF de la maison d'édition pour la Jeunesse dans les années 1960, vice-président de l'Union des écrivains, actif dans la Société européenne de la SF dans les années 1970-1980, importateur (depuis le français et l'italien) et exportateur (vers la France et la Belgique notamment) d'écrits du genre, en est un exemple. En Bulgarie, les positions détenues par les défenseurs du genre sont moins prestigieuses et leurs ressources internationales plus circonscrites : l'Union des écrivains ne comptera qu'un seul prosateur de science-fiction se revendiquant de cette appartenance, Ljuben Dilov, un auteur multipositionné dans le champ littéraire bulgare où il figure dans les jurys des principaux concours d'écriture SF, les comités de 
rédaction des grandes revues et la direction (collégiale) de Galaktika. Si son rôle de protecteur des clubs est relayé par quelques critiques, les grandes figures de la littérature socialiste, y compris lorsqu'elles abordent le registre fantastique ${ }^{52}$, refusent de se muer en porte-parole d'un genre que d'aucuns considèrent avec condescendance, tout en enviant sa popularité et ses tirages insolents. Les soutiens viennent plutôt du monde des revues de science-fiction dont les éditeurs en chef cumulent les profils de scientifiques et lettrés, écrivains à leurs heures ${ }^{53}$.

De fait, la non-synchronisation des horloges terrestres bulgare et roumaine a pour pendant des découpages contrastés de la voûte céleste. Lorsque les premiers clubs voient le jour, la Roumanie, qui a pris ses distances avec l'Union soviétique à partir de 1964, s'ouvre progressivement vers l'Ouest. Les promoteurs de la science-fiction nationale s'insèrent dans les réseaux internationaux en cours d'institutionnalisation à une échelle européenne à la fois façonnée par et transcendant le clivage Est-Ouest. Ils mobilisent les canaux de la francophonie et de la " latinité ", tissant des liens avec des auteurs français, italiens et belges tout en promouvant une définition « littéraire » et « européenne » - non américaine - de la SF. La Bulgarie, elle, aborde la science-fiction depuis les mondes de la critique intérieure, au premier chef soviétique, mais aussi est-européenne (polonaise, est-allemande, tchécoslovaque et hongroise ; yougoslave parfois dans les années 1980). De ces spatialisations distinctes, des périmètres du permis également, attestent les appellations données aux clubs. Si elles témoignent d'une fascination partagée pour le cosmos, le futur et la science, vecteur de rêve ainsi que d'une commune mobilisation de références tutélaires liées aux fiertés scientifiques nationales, les clubs bulgares s'ancrent davantage en terre soviétique (Efremov, Strugackij, Andromeda, etc.), éventuellement polonaise (S. Lem) ou optent pour des appellations "politiquement" neutres (xixe siècle). Les cénacles SF roumains se revendiquent d'une cartographie globale, aussi bien d'un Est non soviétique (le cénacle Solaris de Bucarest) que de l'Ouest, le club de Timişoara s'autorisant la liberté de prendre le nom de H.G. Wells, auteur investi comme " socialiste».

Ces territorialisations du ciel reflètent des politisations distinctes du futur. Au-delà de la diversité des sensibilités individuelles, aux yeux des initiateurs des clubs bulgares, la scrutation du cosmos doit servir une interrogation sur les moyens de réformer l'ordre socialiste, non de l'abolir ${ }^{54}$. L'un des fondateurs du principal cercle bulgare, Atanas Slavov, a justement résumé la posture défendue dans les années 1970 : «Entre les membres du club, de main en main circulaient des romans comme 1984, Brave New World et Nous. Mais aucun membre ne se tenait pour un dissident politique - ils ne jugeaient pas pertinents de remplacer l'illusion du futur "bonheur communiste" par le futur "bonheur capitaliste". Ils les définissaient comme également nuisibles $»^{55}$. La centralité de la figure d'Ivan Efremov dans l'univers des clubs ne s'explique pas autrement : la trajectoire de cet humaniste qui se consacra à l'exploration du cosmos autant qu'à celle des marges de la terre, autorise une continuité entre science et écriture fictionnelle ; certains louent en lui une pensée sociale du temps visant à définir les coordonnées d'un avenir communiste heureux; d'autres saluent l'esprit encyclopédique; d'aucuns soulignent son rôle de passeur dans la sensibilisation au yoga et la recherche d'une personnalité harmonieuse. Au moment où ils décident de donner son nom à leur cercle, plusieurs fondateurs du Club Efremov sont par ailleurs conscients que l'écrivain, autrefois cité par Jurij Gagarin comme une source d'inspiration, est tombé en disgrâce et vit assigné à résidence. 
31 Cette configuration n'empêche pas les clubs bulgares et roumains d'accueillir de longues discussions autour de futurs pluriels : le futur des sciences, envisagé dans une approche scientiste convaincue de la possibilité d'une planification des destinées humaines ; un futur modèle social de l'humanité, fondé sur la recherche de l'harmonie et une éthique de la lutte contre les inégalités ; un futur entouré de mystère, fruit d'une œuvre humaine à portée philosophique. Ces représentations des lendemains se cristallisent en même temps au contact de productions venues d'ailleurs. Encouragés par les États, déployés par les acteurs du champ littéraire, des transferts s'opèrent, le passage entre les langues se faisant instrument de consécration ${ }^{56}$.

\section{Les circulations internationales : Si loin, si près de l'URSS ?}

32 Au cours des années 1940-1950, la Bulgarie comme la Roumanie connaissent une phase partagée de soviétisation/russification culturelle et linguistique et un redéploiement consécutif des lieux, acteurs et supports de circulation internationale du livre. Cette orientation s'estompe toutefois en Roumanie dans les années 1960, laissant émerger une stratégie de nationalisation par internationalisation. En dépit d'une progressive ouverture vers l'Ouest de l'Europe et, de manière plus timide encore, vers les espaces transatlantiques, en Bulgarie le principal référent symbolique, pôle de rayonnement et partenaire demeure l'Union soviétique. L'on aurait toutefois tort d'y voir le marqueur d'un ancrage à l'Est ou d'une autodéfinition « orientalisante » des identités culturelles privilégiées : le lien à l'URSS vaut comme médiateur de l'accès à l'international et sert une revendication d'européanité et de modernité. Quoique par des cheminements différents, Bulgarie et Roumanie se retrouvent donc engagées durant la décennie 1970 dans des entreprises de revendication d'identités croisées : nationale, européenne et socialiste. Un bref retour sur les importations de la science-fiction internationale et les stratégies de promotion des écrits vernaculaires à l'étranger éclairera cette dynamique.

Qui s'emploierait à dessiner la cartographie des traductions en bulgare et en roumain verrait s'esquisser la superposition de paysages semblables, au-delà de quelques « blancs». Les années 1960 accompagnent la traduction et la diffusion massive des grands noms de la science-fiction soviétique (dont certains, on l'a dit, ont été édités dès la seconde moitié des années 1940) : les frères Strugackij, Ivan Efremov, Aleksandr Beleaev, Il'ja Varšavskij; les porteurs de la renommée occidentale se frayent également un large chemin dans les revues et collections (H.G. Wells, Ray Bradbury, Arthur Clarke, Isaac Asimov, etc.) ; les auteurs jugés critiques du capitalisme obtiennent une exposition toute singulière (Robert Sheckley et Clifford Simak par exemple). Certains écrivains connus bénéficient de rééditions de volumes d' « œuvres choisies ». D'autres (ainsi d'Aldous Huxley et de Zamjatin) n'obtiendront jamais de sanction officielle ou verront les écrits tronqués (y compris S. Lem). La présence des plumes est-européennes reste, elle, rare, à quelques exceptions près, comme le Tchèque Karel Čapek ou le Polonais Lem.

À ce segment commun de la topographie littéraire, Bulgarie et Roumanie accèdent par des canaux distincts : jusqu'à la fin des années 1960, c'est dans des traductions réalisées depuis le russe que les lecteurs bulgares goûtent les écrits de Clarke et de Lem dans la revue Kosmos. Bien que les passages depuis l'anglais et le polonais deviennent plus fréquents au cours de la décennie suivante, le russe demeurera jusqu'à la fin de la période le médiateur clé. Non sans une part de tactique : responsables de revues et de 
collections y voient le moyen d'obtenir l'agrément des autorités culturelles à la parution d'écrits occidentaux (puisqu'ils ont déjà été publiés en Union soviétique).

À compter du milieu des années 1960 en revanche, les lecteurs roumains accèdent à une palette de récits qui resteront peu familiers des lecteurs bulgares, à savoir les sciences-fictions italienne et française : la fréquentation roumaine de François Bordes (alias Francis Carsac), Robert Merle, Gérard Klein n'a pas d'équivalent en Bulgarie. Seul Pierre Boule, peut-être, irrigue à parts égales les deux rives du Danube. L'affirmation d'une troisième voie diplomatique s'accompagne en outre, en Roumanie, de manière croissante à partir du milieu des années 1960, d'un déclin des traductions depuis le russe. Indépendamment du renforcement du poids de la science-fiction nationale, leur présence varie peu en Bulgarie : jusqu'à la fin des années 1970, les récits soviétiques publiés dans Kosmos représentent plus d'un tiers de titres, suivis par les textes bulgares (un peu moins d'un tiers), des classiques occidentaux (anglais et/ou américains) et un éparpillement de rares productions autres (japonaises notamment). Dans les deux pays cependant, les années 1980 offrent une diversification des lectures américaines (John Brunner, Frederik Pohl, Algis Budryss) et britanniques (Brian W. Aldiss, H. W. Franke, F. Herbert).

Les circulations internationales des auteurs et les exportations suivent des chemins semblables. Du point de vue des dirigeants et des acteurs culturels des deux pays, la participation à des festivals et congrès internationaux comme la traduction en langues étrangères relèvent simultanément d'une diplomatie culturelle et d'une politique des honneurs ; aux auteurs, elles offrent une ressource capitalisable en interne. Là où la priorité roumaine va à une sortie de l'orbite soviétique que médiatise la langue française, principal vecteur de l'accès à la SF jusqu'au début des années $1980^{57}$, en Bulgarie les élites dirigeantes font le choix d'une insertion internationale adossée à l'URSS. Dans les années 1970 en particulier, la stratégie roumaine s'avère payante ; les bénéfices bulgares sont plus modestes, même si cette orientation extérieure offre aux amateurs de SF l'opportunité d'inviter dans leurs clubs de grands noms soviétiques (ainsi d'Arkadij Strugackij en 1979) en un temps où la petite Bulgarie fait figure, aux yeux de ces derniers, d'espace de liberté - de surcroît fort bien approvisionné - au regard de leur patrie brejnévienne.

En 1972, la première convention européenne de Trieste voit ainsi s'opérer un rapprochement entre Italiens, Français et Roumains autour d'une définition "littéraire» de la SF opposée à l'approche comics des auteurs américains. L'institutionnalisation d'une Europe de la SF serait elle-même le résultat d'une initiative roumaine lancée par Ion Hobana pendant le premier Worldcon de Heidelberg en 1970 aux participants " latins» (France, Belgique, Espagne, Italie, Portugal et Roumanie $)^{58}$. La première participation d'auteurs bulgares à Eurocon date, elle, de 1976. La convention se tient pour la première fois dans un pays est-européen, en l'occurrence la Pologne. Tardive, cette présence est porteuse de dividendes internes : Ljuben Dilov, primé, sera ultérieurement traduit en anglais et allemand. Par la suite, seuls les congrès internationaux organisés à l'Est ou dans la Yougoslavie non alignée accueilleront des Bulgares. En février 1989, la Bulgarie sera ainsi représentée en force (33 participants) à un premier Soccon que fréquente seulement une poignée de Roumains.

Le rayonnement extérieur obtenu par la SF roumaine favorise une certaine diffusion internationale de la littérature vernaculaire par des canaux dits officiels (pour reprendre la terminologie de Ioana Popa), soit les milieux d'éditeurs et d'agences 
tissant des relations avec des partenaires étrangers. La piètre présence des écrivains bulgares dans les réseaux professionnels internationaux éclaire quant à elle la rareté des contacts directs avec des éditeurs ${ }^{59}$. L'exportation des œuvres y relève d'une diplomatie culturelle d'état et de ses agents, l'éditeur en langues étrangères Sofia Press et l'Agence Jusautor. $\mathrm{Au}$ moment où la libéralisation s'accompagne d'une nationalisation du communisme, la traduction des grandes épopées patriotiques est prioritaire. ${ }^{60}$

39 Dans les années 1980, l'ensemble des coordonnées relatives à la production et à la diffusion des sciences-fictions locales est redistribué : à l'ouverture de la décennie 1960-1970 succède en Roumanie un national-communisme autarcique, hanté par le remboursement de la dette extérieure et la dégradation des conditions de vie ; confronté à une crise moins dramatique, le régime bulgare s'effile lentement, trahissant les espoirs d'une génération diplômée qui aspire à vivre ici et maintenant et qui contemple impuissante le repli conservateur d'un Todor Živkov vieillissant. En Roumanie, lectures sciences-fictionnelles et univers des clubs offrent de plus en plus souvent une échappée hors du temps; en Bulgarie, la SF gagne de nouveaux passionnés, au-delà de la jeunesse plutôt masculine qui avait jusqu'alors fait sa fortune, les cercles de SF devenant l'un des lieux où sont débattues des pensées iconoclastes, poétiques ou politiques.

\section{La fin des années 1980 : un avenir immobile?}

Au soir descendant des années 1980, les articulations entre le temps des écrivains, le temps de lecteurs et les rythmes circulaires d'une vie politique ossifiée virent à la dissonance. Les mondes de la science-fiction dépeignent désormais la disjonction entre générations, dans la société comme les arts. Adolescents et étudiants rêvent de la remise en marche d'un temps dont l'accélération officiellement proclamée ne s'accomplit plus, tout en redoutant l'avenir. De part et d'autre du Danube gris, l'aspiration à un ailleurs se mêle à l'extension des séductions de l'ésotérisme et des contes et légendes vernaculaires. Avec l'accession au pouvoir de Gorbačev en 1985, temporalités bulgare et roumaine évoluent toutefois en des directions opposées : dans le contexte roumain, l'aggravation des pénuries jouxte la réitération ad nauseam des images de Ceauşescu et des accomplissements vertigineux de "son époque d'or ". En Bulgarie, la perestroïka soviétique est suivie avec fébrilité tandis que, dans les clubs, s'imagine un monde au-delà de la partition entre socialisme et capitalisme.

\section{De l'apocalypse aux profondeurs mythologiques de la nation}

41 Au tournant de la décennie 1970-1980, le futurologue soviétique Igor Bestužev-Lada avait noté l'avènement à l'Ouest d'une nouvelle ère d'alarme, sur fond de "Seconde Guerre froide $»^{61}$. L'observation vaut également pour l'Est. Souvent dus à des trentenaires ou quadragénaires, dont certains, à l'instar d'Agop Melkonjan en Bulgarie, sont influencés par le cyberpunk occidental, les textes, majoritairement dystopiques, dépeignent un futur étiré jusqu'à la distorsion, que ce soit vers un avant postapocalyptique ou un passé aux insondables profondeurs. L'aujourd'hui glisse quant à lui dans l'absurde : "Nous vivons sans aucune perspective. Ni aucun espoir. Et pourtant, beaucoup d'hommes vivent ainsi... Sans rien espérer, sans rien attendre ", 
écrit dans son mini-roman Anomia (L’anomie, 1986) le jeune Mihail Grămescu ${ }^{62}$, primé au Soccon de 1989. Constantin Cozmiuc intitule l'un de ses récits " Le temps est notre ombre » $(1987)^{63}$. La crise de l'industrialisation socialiste à cadences élevées, la prise de conscience de ses conséquences environnementales et les échos de la catastrophe nucléaire de Černobyl nourrissent cet imaginaire d'un désastre imminent. Face à l'optimisme de commande ressurgit l'inquiétude devant la science et, avec elle, une tradition littéraire européenne alliant progrès technique et violence.

Le creusement de la distance avec le présent ne concerne pas les seuls lendemains : maints récits et romans viennent envelopper leurs intrigues d'un manteau aux couleurs des contes populaires nationaux, des mythes antiques, voire des références bibliques. Dans les années 1970, confrontés à l'éloignement progressif du bonheur collectif qui semblait une décennie plus tôt à portée de vie, les régimes communistes ont entrepris une politique de ressourcement dans l'histoire nationale visant à ancrer les destinées socialistes et la révolution technico-scientifique en un passé autochtone à travers une valorisation des origines indigènes, thraces en Bulgarie, daces en Roumanie ${ }^{64}$. Le "protochronisme", critique radicale de l'eurocentrisme et affirmation d'une supériorité nationale sur la longue durée, clive le champ culturel roumain tout en légitimant le national-communisme. En Bulgarie, le processus d'antiquisation de la nation atteint son apogée lors de la célébration du $1000^{\mathrm{e}}$ anniversaire de la création d'un État « bulgare » (1981) : de dispendieuses fictions sont produites sur commande, afin de doter le futur socialiste d'antécédents glorieux.

Cette exploration du sillon national suscite l'adhésion de larges segments des intelligentsias bulgare et roumaine, ne serait-ce que parce qu'elle offre initialement une échappée par rapport aux canons thématiques et esthétiques du réalisme socialiste. Les mondes de la SF saisissent en outre cette opportunité pour conforter leur inscription dans les traditions littéraires autochtones, notamment en publiant des auteurs de l'entre-deux-guerres. Avant même le cours nationaliste, les hantises de l'histoire nationale avaient occasionnellement habité les textes SF, comme en témoignent Trimisul dacilor [L'envoyé des Daces] de Mihu Dragomir (1964) ou Galeteja [Galatée] de Petăr Bobev (1967). Situé à la fin de l'Empire ottoman, ce récit creusait les entrailles d'une mer fantastique pour mieux relire (et corriger) une histoire de souffrances sous le " joug turc ». L'inflexion patriotique encourage encore davantage l'identification entre la terre mère et la longue durée des consciences nationales, particulièrement dans les écrits à destination de la jeunesse ${ }^{65}$.

De fait, la nationalisation des socialismes bulgare et roumain ne procède pas seulement à la valorisation des inimitiés nationales structurantes : elle s'accompagne aussi d'une double opération d'antiquisation et de relocalisation du futur ${ }^{66}$. De la place croissante de l'exhumation du passé dans le façonnage du rêve atteste, par exemple, l'évolution du contenu et de l'iconographie de la très populaire revue bulgare Kosmos : à partir des années 1970, dans cette publication conjuguant vulgarisation de la science, grandes découvertes et récits de SF, l'exploration de l'Ailleurs, autrefois confiée à la lumineuse conquête spatiale, se déplace vers une quête des origines où l'archéologie semble recevoir pour mission de penser aussi bien l'enracinement de la nation que sa projection dans l'avenir. Par un étonnant jeu sur les espaces-temps, un lien est désormais instauré entre l'« en-dessous » (les profondeurs de la terre) et l'« au-delà » (cosmique). Corrélativement, les couvertures de la revue glissent entre deux voûtes célestes des références aux héros nationaux. Ainsi, le visage éternellement adolescent 
de Vasil Levski, révolutionnaire tué en 1876, orne-t-il la première page d'une livraison de 1987, à parité avec une étoile brillante. Entre les deux images est imprimé le texte suivant : « Le temps est avec nous et nous sommes dans le temps", nation et cosmos entretenant désormais des relations de symbiose.

En Roumanie, tandis que les conférences nationales des clubs de science-fiction ne manquent plus d'inclure dans leur programme des années 1980 un panel sur le développement technique et scientifique des Daces à la lumière des nouvelles découvertes archéologiques, certains récits de science-fiction confèrent aux voyages dans le passé une nouvelle coloration, régionale et ethnoculturelle. Mircea Oprị̧ă, représentant la génération ayant débuté dans les années 1960, publie dès 1973 une nouvelle, "Une faille dans le temps", opérant un retour dans l'espace-temps des Carpates transylvaines ${ }^{67}$. Il y dépeint un voyage initiatique sur des terres de nostalgie, à des milliers d'années en arrière, et l'accident survenu lorsque, s'engouffrant dans une faille dans le temps, père et fils se retrouvent encerclés d'hommes en uniformes : là où le père entend parler allemand "schmutziger Rumäne" «Los, los", le fils croit déchiffrer du russe " Rumjanskij špion », « davaj, davaj ». Les deux héros n'en finissent pas moins par échapper à l'étreinte de cette occupation " allemande et/ou russe " pour retrouver l'espace de leur indépendance.

La nouvelle de M.Oprị̧ă met en exergue une autre évolution sensible dans les thématiques des années 1970, et plus encore, de la décennie suivante, la (dés-)union entre pères et fils. Dans ce temps collectif dont le futur s'épuise dans un avenir inaccessible ou dans la conscience d'une ancienne fatalité, la transmission par la filiation devient improbable. Maints récits bulgares et roumains tissent des intrigues où les héros repoussent la ressemblance avec les pères et tentent d'échapper à leur ombre au prix d'une certaine marginalité sociale. Ariana, première nouvelle du jeune Nikolaj Vatov, dépeint ainsi les fils privés de références paternelles : dans ce monde du futur où même les navettes spatiales ont du retard, un jeune homme croise un ancien pilote, en un bref interstice de temps. De la mission spatiale effectuée par celui-ci, les traces ont été effacées, interdisant le tissage d'un lien intergénérationnel par le truchement $\mathrm{du}$ souvenir ${ }^{68}$. Alors que la confrontation avec le paradoxe temporel vaut anéantissement, des " orphelins » commencent à peupler les textes de jeunes auteurs $\mathrm{SF}^{69}$.

Désinsérés de toute lignée, les individus que les années 1960-1970 avaient arrachés à la servitude du collectif se retrouvent désormais étonnamment seuls. En 1988, la revue FEP décerne son second prix à un récit d'abandon, « Il fait nuit». Quelque part, en un " bloc » immense situé hors temps, un cri déchire le silence ; ses échos éparpillés se heurtent aux murs du bâtiment et à une absence de réponse : " - Qui a dit "Venez" ? Personne. Alors la voix lasse se rétracta dans mon corps replié $»^{70}$. Révélateur de ce désenchantement est également le courrier adressé à l'écrivain de SF, Agop Melkonjan, par un débutant primé au concours de la centrale nucléaire de Kozlodui. Invité à se présenter, l'auteur écrit : « Je suis né le 16 juin 1955 à Sofia. J’ai effectué mes premières tentatives de création à l'âge de 14 ans, avec de la poésie ». À la missive, il a joint un feuillet séparé : " pour être franc, j'ai éprouvé d'immenses difficultés à écrire cette mienne "biographie". Je place le terme entre guillemets car je ne vois rien dans ma vie qui puisse mériter d'être décrit. Sous quelque angle que je la regarde, je ne vois qu'une succession infinie de peines». De cette seconde lettre, Agop Melkonjan décide de 
reproduire des extraits et commente, paternel et impuissant, le récit d'« un homme pour qui le mouvement a toujours été une source de souffrance $»^{71}$.

Assurément, tous les lecteurs n'ont pas perdu leurs appétences pour le futur. Une enquête conduite dans le milieu des clubs bulgares en 1987 suggère que celles-ci sont avant tout générationnellement stratifiées : à la question " Où iriez-vous si vous disposiez d'une machine à voyager dans le temps ? », près de la moitié des présidents de clubs répondent qu'ils préféreraient explorer le passé, un cinquième seulement choisissant le futur. Plus jeunes, les adhérents des clubs demeurent fascinés par l'avenir (63\%), seuls $18 \%$ préférant remonter le temps. Indépendamment des appartenances générationnelles, les ères lointaines exercent néanmoins une séduction partagée : « Le lien présent-futur est brisé, concluent les auteurs de l'enquête ; la dépendance entre les événements présents et leurs conséquences futures est en train de se perdre $»^{72}$. Sur ces expériences sociales différenciées, l'univers des clubs ouvre une fenêtre donnant à voir un kaléidoscope d'émotions et d'attentes éclatées.

\section{Les mystères du monde des clubs et les marches du temps}

49 En cette étrange décennie de lassitude politique et d'hivers froids, les clubs accueillent des amitiés parfois tendres, des échanges de disques, VHS et cigarettes à la lisière de l'illicite et des discussions étoilées de volutes de fumée. Au-delà des différences de contexte politique, les fans de science-fiction bulgares et roumains se rejoignent pour fonder leur croire individuel et collectif en l'avenir dans une science plus que jamais abordée à travers ses mystères. Combien semble éloigné le temps où la science, toute de rationalité et d'objectivité, s'identifiait aux grandes enjambées des progrès terrestres ou stellaires ; de proche en proche, s'estompe la ligne de discontinuité entre la passion des explorations scientifiques et les séductions du surnaturel et du paranormal.

Plusieurs facteurs éclairent la manière dont, sous le socialisme tardif, la fascination pour la parapsychologie, l'hypnose ou l'ésotérisme s'instille dans les mondes de la science-fiction. Le premier plonge ses racines dans une sensibilité à l'occultisme et à la cosmologie cristallisée au tournant $\mathrm{du} \mathrm{xx}^{\mathrm{e}}$ siècle dans les milieux littéraires symbolistes et l'art pictural européens. À l'époque, celle-ci avait connu une large réception dans les cultures russe $\mathrm{e}^{73}$, bulgare et roumaine. De retour d'un séjour initiatique de trois ans à Calcutta en 1931, Mircea Eliade avait ainsi consacré plusieurs ouvrages au yoga et à la spiritualité indienne, rapprochée de la spiritualité roumaine ${ }^{74}$. Dans les années 1920-1930, puis de nouveau dans les années 1950-1960, les possibilités offertes à la pensée utopique par le développement de la science ont conduit, en particulier en Union soviétique, à réactiver ces imaginaires : « Lorsque la science s'est fondue avec la pensée utopique, note très justement Birgit Menzel, et lorsque, pendant "l'ère cosmique" proclamée, les frontières entre la science et la fiction fantastique se sont déplacées, certaines disciplines telles que la télépathie, l'hypnose et la parapsychologie [...] ont connu un développement exponentiel $»^{75}$. Ce brouillage entre science, parasciences et fiction, diffusé à travers les revues de vulgarisation scientifique soviétique $^{76}$ et leurs homologues locaux, n'affecte alors pas seulement le «bloc soviétique ": en Amérique aussi le désir de mieux maîtriser le fonctionnement du cerveau humain, notamment dans le cadre de la préparation des cosmonautes, et de la compétition scientifique avec l'URSS tendent à distendre les contours du champ scientifique ${ }^{77}$. 
51 Au tournant des années 1970-1980, de nouvelles circulations Est-Ouest s'observent : avant même que l'œuvre du mystique théosophe bouddhiste, Nikolaj Rerih, russe installé aux États-Unis entre les deux guerres ${ }^{78}$, ne soit redécouverte en URSS, Ljudmila Živkova, la fille du dictateur bulgare, Todor Živkov, en fait un médiateur de son intérêt pour l'ésotérisme, le yoga et les philosophies orientales ${ }^{79}$. Présidente du Comité à la culture entre 1975 et son décès prématuré en 1981, elle lui consacre l'un de ces immenses programmes culturels d'État qui constitue sa marque de fabrique, le "Programme Nikolaj Rerih », étape d'un " socialisme mûr " souhaitant faire cultiver en l'être sensibilités culturelles et spirituelles ${ }^{80}$. En Roumanie, dès la fin des années 1960, les pouvoirs tentent, sans succès, de convaincre Mircea Eliade, professeur à l'université de Chicago partageant sa vie entre les États-Unis et la France, de revenir sur sa terre natale. Peu à peu, ses récits et nouvelles fantastiques retrouvent une place, certes parcimonieuse, dans le paysage littéraire local, notamment les anthologies d'anticipation ${ }^{81}$. Son Traité d'histoire des religions est quant à lui publié dans les années $1980^{82}$. Les lecteurs s'alignent dès l'aube en longues files d'attente pour se procurer le dernier volume de l'ancien apprenti yogi, fasciné par l'ésotérisme et la magie, ayant accédé à une visibilité mondiale par le renouvellement de l'histoire des religions.

Le goût pour l'inexplicable dans les sciences reflète sans doute, en second lieu, une aspiration partagée à discuter certains enjeux métaphysiques que la religion orthodoxe avait pris en charge jusqu'à l'instauration du communisme. À la différence d'une Union soviétique où les campagnes d'athéisme furent d'une rare intensité ${ }^{83}$, en Bulgarie et en Roumanie cet investissement du cosmos ne saurait être uniquement envisagé comme une réponse à une crise du croire dans l'efficacité du songe stellaire. Il apparaît plutôt comme l'une des modalités de cristallisation de celui-ci : c'est précisément la capacité à accueillir et à agréger une pluralité d'enjeux relatifs au croire (les origines de la vie, l'horizon des destinées humaines, les territoires de la mort, etc.) qui a fait advenir le rêve cosmique. Dans maints récits fictionnels ou non, dans moult discussions d'amateurs, la pensée bascule souvent du scientifiquement établi, au possible littéraire, voire au croire fondé en mystique : les mondes de la science et de la fiction scientifique y entretiennent des relations de gémellité.

53 À la fin des années 1970, le club Efremov de Sofia propose ainsi un module d'enseignement de 80 heures «La construction de la personnalité créative» qui conjugue yoga, initiation aux philosophies orientales à la "pratique heuristique de l'Institut technologique de Californie ». Son concepteur, Hristo Gešanov, appliquera ultérieurement ce programme dans des lycées pilotes, des usines et même auprès de cosmonautes, sur sollicitation de pouvoirs publics soucieux de maximiser potentialités cérébrales et sensorielles ${ }^{84}$. Le club Efremov ira jusqu'à inviter à ses réunions un célèbre occultiste bulgare (une initiative ayant valu au directeur une "discussion franche" dans les locaux de la police politique ${ }^{85}$ ), tandis qu'à Plovdiv le $V^{e}$ festival de science-fiction d'octobre 1988 organise, entre une studieuse table ronde sur l'enseignement et une soirée de musique électronique, un débat sur les " extra-sens » : la discussion porte sur l'interprétation de l'aura entourant la main d'une voyante qu'une image photographique serait parvenue à capter ; un journaliste de l'hebdomadaire Orbita y propose la création d'un Département « Extra-sens » auprès du nouveau Centre national des médecines non traditionnelles ${ }^{86}$.

54 En Roumanie, outre l'influence de Mircea Eliade, l'on assiste dans les années 1970 à un intérêt grandissant pour la parapsychologie, la télépathie, les biorythmes et les 
médecines alternatives inspirées de thérapies populaires et de courants orientaux. Les techniques de respiration yoga finissent par atteindre certains secteurs du monde hospitalier avant d'être présentées - en tant que sport et " source de santé » assurant le " développement multilatéral » de l'être humain ${ }^{87}$ - dans quelques maisons de la jeunesse ou des syndicats. Les services de renseignement et du ministère de l'Intérieur manifestent leur intérêt. En 1977, un ingénieur roumain émigré à Paris reçoit ainsi l'accord des autorités pour présenter devant des scientifiques "la science de l'intelligence créatrice» fondée sur la " méditation transcendantale ». Cette dernière fait maints émules dans les milieux de l'intelligentsia humaniste et technique de Bucares ${ }^{88}$. En parallèle, des cours de civilisation indienne et de sanskrit sont proposés à l'université de Bucarest, parfois guidés par l'ambition de démontrer la proximité entre la civilisation « thraco-dace » et la civilisation indienne.

À ce tissage de parentés entre rationalité du savoir et imaginations ésotériques, les pouvoirs publics bulgare et roumain apportent des réponses ambivalentes : en Bulgarie, pendant que Ljudmila Živkova cultive une fascination mystique pour le règne du bizarre, la clairvoyante Baba Vanga est érigée en pythie du pouvoir ${ }^{89}$. Les ordinaires citoyens professant des croyances hétérodoxes voient en revanche leurs ouvrages saisis ${ }^{90}$. En Roumanie, l'État engage en 1981-1982 des poursuites sévères contre les adeptes de la "méditation transcendantale" (dénoncée comme secte) et du yoga (interdit comme " manifestation de mysticisme » encourageant la pornographie et les comportements antisociaux); plusieurs hauts responsables politiques en font les frais ${ }^{91}$. Cependant les mystères de la "science à la frontière du savoir" ne disparaissent pas du nouvel Almanah AnticipaŢia, annuel de 340 pages édité par le Comité central des Jeunesses communistes, dédié notamment à la production de clubs ${ }^{92}$. Il n'est d'ailleurs pas à exclure que la conjugaison entre adoubement élitaire et dénonciation publique ait pu participer du rayonnement des courants de pensée ésotérique.

Dans la Bulgarie de la fin des années 1980 l'engouement pour le paranormal est tel que les éditeurs de la revue Kosmos se sentent contraints de suspendre l'une des rubriques préférées des lecteurs, "La cartothèque des cas mystérieux », et d'opérer une mise au point :

Il est vrai que depuis plus d'une année Kosmos paraît sans sa " Cartothèque ». La principale raison en tient au fait que ces derniers temps l'attention des lecteurs s'était révélée unidirectionnelle. La plupart des lettres que nous recevions provenaient de lecteurs partageant leurs observations d'objets volants non identifiés. [...] Chers lecteurs, la rédaction n'a jamais douté de votre bonne foi [...]. Vous affirmez que l'objet lumineux émettait une lumière jaune, votre ami est prêt à jurer que la lumière était bleue. Tout ceci est pleinement compréhensible. Les gens observent des choses similaires, mais les remarquent et se les remémorent différemment [...]. Comme se sont accumulées des lettres aux contenus identiques [...] nous avons décidé de suspendre " La cartothèque des cas mystérieux ». Mais vos courriers ont continué à affluer...93

Après avoir relaté trois cas "mystérieux» d'OVNI, la rédaction, prudente (nous sommes en pleine perestroïka), les soumet à la sagacité de son lectorat tout en invitant celui-ci à diversifier ses « observations ».

Cependant, l'occulte et l'énigme ne constituent ni le seul vecteur d'entrée, ni l'unique sensibilité représentée dans les mondes de la science-fiction. Les clubs représentent plutôt l'un des lieux où s'expose, comme dans un kaléidoscope, l'éclatement générationnel, spatial et politique de sociétés à qui les pouvoirs communistes avaient 
espéré donner un futur unifié, supervisé depuis le centre. Pour certains jeunes, les cénacles demeurent ainsi ce lieu d'affinités électives, de curiosités partagées et d'ouverture sur le monde qu'évoque rétrospectivement Val Todorov, ancien membre du Club Efremov :

À l'époque, Nasko Slavov, en véritable apôtre de la juste foi des rêveurs, discourait de mondes inconnus et de réalités fantastiques sur fond de musique des Pink Floyd tout en nous montrant des diapositives de couvertures de disque psychédéliques et de collages surréalistes. [...] J'ai trouvé d'autres adeptes du culte des frères Strugackij, Lem, Vonnegut et, de là, découvert le réalisme magique de Julio Cortazar, Marquez et Llosa. J'ai découvert la musique de Genesis, des Pink Floyd, de Yes et monté mon premier spectacle multimédia sur « 2112 » de Rush. J'ai trouvé des fans du temple de Tarkovskij, Fellini et Kubrick; nous avons créé un club de cinéma et filmé le premier film vidéo indépendant " La meilleure défense ». J’ai trouvé le courage et l'inspiration pour écrire mes premiers récits et un éditeur pour mon livre ("Irkala, le pays des morts»), le même Nasko Slavov à la jeunesse impérissable. J'ai rencontré des amis de toute une vie. ${ }^{94}$

Un segment de la jeunesse y cultive l'idée de marginalité (tant par rapport aux attentes politiques qu'aux contraintes sociales d'une classe moyenne urbaine dont les règles de bienséance ont été définies par la génération des parents) et le sentiment d'une supériorité (liée à une maitrise des sciences vécue dans l'entre-soi). Avec le renforcement du contrôle politique, dans les cénacles roumains en particulier, la dimension " communauté affective » gagne en importance, elle qui ne suppose pas de confrontation avec le régime - ou alors d'une manière oblique, comme en témoigne cette remémoration :

Marian Gogoaşe, un jeune grand et blond, avec beaucoup de succès auprès des dames et un humour débordant [...] nous présentait périodiquement son roman (" interminable», complétaient les mauvaises langues du cénacle - guère nombreuses) intitulé « La fin du monde ». Comment ne pas aiguiser les oreilles en pleine " époque d'or»? Constanţa Paligora, rédactrice de la maison d'édition Albatros [...] l'a mis en garde [...] : dans l'éventualité d'une publication, ce titre paraîtra aux " camarades" de la censure absolument inadéquat par rapport à l'optimisme communiste. À la réunion suivante, Marian Gogoaşe continua imperturbable à nous lire son texte, qui portait désormais le titre beaucoup plus... éthique « Le début de la fin du monde ».95

59 Les festivals de science-fiction permettent en outre de cultiver la dimension nocturne de l'être ensemble dans la consommation d'alcool et de cigarettes. Entre les lectures de textes de jeunes auteurs et d'écrivains étrangers souvent non publiés localement s'immiscent le visionnage de films (tel 2001, Odyssée de l'espace, au programme de plusieurs conférences roumaines des années 1980 avec un sous-titrage en polonais... ${ }^{96}$ ) et l'écoute de musique ${ }^{97}$. Sur fond de pénuries, les amateurs échangent des livres (photocopiés ou typographiés) et le marché noir des produits SF vient accompagner un marché noir tout court. Le positionnement géostratégique, la proximité avec telle ou telle frontière (Timişoara capte les télévisions yougoslave et hongroise ${ }^{98}$; à Bucarest l'on se prend à regarder la télévision nationale bulgare ; Varna bénéficie de son statut portuaire et des circulations internationales le long du Danube ; l'Ouest bulgare regarde du côté yougoslave, etc.) et la présence d'étudiants étrangers (des pays frères comme des États socialistes du monde arabe et d'Afrique) dessinent une cartographie des liens en décalage avec la lecture officielle. L'espace national homogène apparaît transfiguré au gré des insertions localisées d'économies nationales autarciques (particulièrement celle de la Roumanie) et, en même temps, un peu " globalisées ». 
60 En province, dans les moyennes villes monoindustrielles, l'atmosphère est plus sombre. Socialisés dans les clubs et cénacles des grandes villes universitaires, les étudiants bulgares et roumains sont parfois envoyés exercer leur métier dans des périphéries où le temps est scandé par l'alternance des saisons et, de plus en plus souvent, les coupures d'électricité. Au sein même des clubs, le contraste se creuse entre les espoirs de modernité incarnés par la science-fiction et les possibilités effectives de nourrir l'inventivité. En 1988, les responsables du Club Hronos de Sopot déplorent ainsi la fin d'un « âge d'or » :

J'ai entendu dire que l'époque où les clubs furent créés était plus enflammée, qu'elle réunissait des personnes parentes de pensée, prêtes à leur consacrer l'intégralité de leur temps libre sans formuler de prétentions démesurées en matière d'aménagement ${ }^{99}$.

61 Derrière le propos sourd l'inquiétude des présidents des clubs (bulgares plus que roumains) devant la distance qui les sépare d'une nouvelle génération soupçonnée de préférer à l'écrit la séduction d'images filmiques (d'horreur notamment) et de se laisser subvertir par un matérialisme que leurs aînés, critiques de l'embourgeoisement socialiste, avaient tendu à rejeter. En 1988, Atanas Slavov publie dans la revue FEP une lettre ouverte où se reflètent ces attentes contraires :

« Lettre ouverte au fan bulgare»

Qu'es-tu aujourd'hui fan bulgare?

Est-il vrai

Que tu ne cherches plus l'étrange ;

Que tout ce que tu attends de la science-fiction aujourd'hui est qu'elle te fasse peur ou sourire ;

Que le « chewing gum-vidéo pour les yeux » t'a détaché des livres

Que tu ouvres les « fenêtres du futur » seulement pour y voir les cauchemars de ton propre inconscient ;

[...]

Je redoute que l'automatisation du quotidien ne te conduise à oublier la spirale du développement.

Je redoute les gens - qui ont arrêté leur montre ;

Leur science-fiction prédatrice et gâtée, qui prêche l'impuissance devant une histoire itérative ;

Leur cynisme autosatisfait qui remplace les « fenêtres de l'avenir » par un miroir reflétant leur propre Ego.

Je redoute, mais j'espère également

Que le doute envers les autorités libérera ta pensée ;

Que de tes lignes écrites naîtront... non les nouveaux Clarke et Efremov, Sheckley et Strugastki, mais d'autres œuvres inimitables qui joueront un rôle démiurgique dans l'époque nouvelle [...]

Parce que la science-fiction est le prolongement de la poésie par d'autres moyens. Je tente...

Je crains...

J'espère... ${ }^{100}$

62 Que la jeunesse s'occidentalise sans que les gardiens du goût et de l'authenticité nationale ne parviennent à prévenir cette évolution constitue une menace jumelle, évoquée en 1988 par Petja Dimitrova, membre du jury du concours de FEP, dans ses conseils aux jeunes écrivains :

Il n'est pas obligatoire, dans vos manuscrits, d'emprunter au récit fantastique américain de masse, très inégal, aussi bien dans votre style que dans votre syntaxe qui sonne souvent comme une piètre traduction, jusque dans les noms des héros. On en ressort avec l'impression que dans le futur n'évolueront que des personnes 
portant des noms anglo-saxons, comme si les Slaves n'étaient pas allés les premiers

dans l'espace, comme si la Bulgarie ne faisait pas partie des pays cosmiques. ${ }^{101}$

63 roumain l'arène d'une lente déliquescence des régimes socialistes par infusion de repères culturels occidentaux et développement d'une société de consommation. Si l'existence des clubs a pu participer d'une remise en question de l'ordre communiste, c'est en effet à travers un autre canal : leur capacité à accueillir une pluralité d'ambiances, d'univers sociaux et d'usages de la science (fiction). Cette diversité venait en effet démentir la capacité des pouvoirs publics à imposer une narration univoque de la triade passé-présent-futur. Dans le contexte roumain, les cénacles ont ainsi accueilli la résurgence de débats sur le rapport à la nation et les inscriptions géoculturelles des mondes lettrés : les hommes de culture se sont définis par rapport à une génération de l'entre-deux-guerres, clivant " pro» et « antioccidentaux». Un segment des intellectuels polyglottes a eu en outre le sentiment que le communisme avait promu de faux nouveaux intellectuels ingénieurs, dont le genre de la science-fiction lui-même portait certains des stigmates. De leur côté, les « ingénieurs auteurs » ont emprunté le chemin de la SF pour entrer dans un monde littéraire fermé sur ses certitudes.

En Bulgarie, où les lignes de partage entre les milieux lettrés urbains et les diplômés du technique étaient sans doute moins profondes et ne recouvraient pas des oppositions entre Est et Ouest, les débats sur le futur cosmique ont également épousé une variété d'options politiques et éthiques, mais celles-ci sont demeurées unies dans une commune affirmation de la supériorité de la libre-pensée sur une tatillonne bureaucratie. L'intellectuelle Miglena Nikolchina qui, sans se revendiquer du label "science-fiction» a publié dans maintes revues et anthologies du genre, a justement restitué cette atmosphère des années 1980 :

En tant qu'hybride entre la " science » et la " fiction », entre les sciences et les humanités, la science-fiction fournissait un exemple et un pont pour s'affranchir plus avant du contrôle politique sur les humanités, la littérature et les arts. [...] la science-fiction était interdisciplinaire, transférable et traduisible ; elle n'était liée à aucune identité particulière ; elle pouvait traverser les frontières ; il était possible d'y entrer depuis différentes positions politiques, disciplinaires et personnelles ; elle était interactive et ouverte au débat. Elle exprimait une insatisfaction envers ce qui était. ${ }^{102}$

De cet éventail des orientations, le $\mathrm{V}^{\mathrm{e}}$ festival de science-fiction de Plovdiv offre une étonnante captation photographique : là où les responsables du Club de la ville danubienne de Svištov (sensibles aux enjeux de pollution industrielle) évoquent leurs discussions avec les organisations écologistes informelles et le Club pour la perestroïka et la transparence (principaux vecteurs d'une opposition au régime communiste), ceux de Plovdiv décrètent que, profitant de la nouvelle réglementation sur l'initiative économique privée, ils ont l'intention de transformer leur club en coopérative et de développer des activités profitables ${ }^{103}$. En cette année 1988, le temps semble prêt à se remettre en mouvement. Et de ce mouvement qui allait bientôt porter certains responsables de clubs à s'engager aux côtés des forces " anticommunistes » et d'autres à rester dans les rangs du parti communiste réformé, les cercles de science-fiction ont été l'une des courroies. 


\section{Conclusion}

66 L'exercice qui consistait à prendre pour terrain d'observation un objet minuscule, des auteurs et intrigues livresques s'interrogeant sur l'avenir individuel et collectif, pour en faire le support d'une réflexion sur l'enchâssement entre les temporalités d'un genre littéraire, les productions politiques du temps et les représentations sociétales des relations passé-présent-futur était confronté à plusieurs écueils. En la matière, le désir était de ne pas postuler une surdétermination politique de la science-fiction en Bulgarie et en Roumanie, ni d'envisager celle-ci uniquement par le prisme de l'autonomie littéraire ; il était aussi d'observer un monde d'entre-deux qui n'évolua pas en dehors des temporalités politiques, mais ne se réduisit pas à elles, les éventuelles accroches étant médiatisées par un ordre des sensibilités. L'exploration des univers science-fictionnels a offert plusieurs prises sur le temps à travers le contenu des œuvres, l'inscription des auteurs dans le champ littéraire, ainsi que les réceptions des textes par des lecteurs dont les quotidiens de loisir et de science en furent partiellement façonnés.

67 La démarche retenue a également permis de recontextualiser les socialismes est-européens en les replaçant dans une configuration temporelle globale - Est et Ouest embrassés - qui fut sans précédent autant qu'éphémère. Les calendriers de l'enchantement et du désenchantement observés en Bulgarie et en Roumanie pourraient aisément être décrits dans les termes de Reinhart Koselleck relatifs au décalage croissant entre « champ d'expérience » et " horizon d'attente » ${ }^{104}$. L'entrée par la science-fiction aide cependant à penser l'une des singularités des ordres socialistes centre-européens, à savoir qu'ils se déployèrent dans une petite fenêtre de temps postérieure à la Seconde Guerre mondiale, où l'accessibilité inédite du ciel ouvrit aux ambitions humaines un espace de rêves difficilement concevable aujourd'hui. À la différence de l'Union soviétique où le socialisme avait d'ores et déjà accueilli deux générations de promesses, de mutations et de violences lorsque le premier homme s'envola dans les cieux, en Europe centrale et orientale les ambitions prométhéennes d'une métamorphose des quotidiens furent scandées en synchronie avec l'élan de la raison scientifique des navettes spatiales. Si le rêve cosmique n'a pas revêtu en Bulgarie et en Roumanie les aspects de projections de puissance globale et de renforcement d'une fierté nationale auxquels il fut associé en URSS, cette chronologie spécifique, reflétée dans les mondes de la science-fiction, a conféré une tonalité originale à la production des lendemains socialistes locaux.

68 La délocalisation spatiotemporelle a par ailleurs mis en lumière les cheminements étranges qui mènent du différent au même, et inversement. Entre la Bulgarie et la Roumanie, plus souvent rapprochées dans les études sur le postsocialisme que le communisme, entre deux États qui, dans les imaginaires sociaux de leurs dirigeants, n'ont jamais constitué l'un pour l'autre des significant others, des parentés se sont dessinées, indépendamment des traversées humaines ou livresques du Danube. Réverbérées dans les intrigues science-fictionnelles et les débats des clubs, ces proximités ont pu concerner, différemment selon les époques, les attentes sociétales, les expériences de la mobilité sociale, les transmissions intergénérationnelles et les croyances ou déceptions politiques. La mise en regard de ces terrains pourrait dès lors ouvrir sur une réflexion sensible aux ressemblances morphologiques au moins autant qu'aux transferts culturels. À bien des égards, ces deux pays dont l'édification 
stato-nationale avait été portée par un espoir de modernisation-européanisation dans la seconde moitié du XIX ${ }^{\mathrm{e}}$ siècle, ont sous le socialisme tardif tenté de négocier, à partir de ressources linguistiques, culturelles et sociales partiellement antérieures au communisme, les attaches spatiales et symboliques leur permettant de poursuivre ce projet. Et ce sont souvent par des voies de traverse qu'ils ont, séparément, produit des intelligences communes

des défis afférents à cette ambition européenne.

\section{NOTES}

1. Pour des raisons pratiques nous utiliserons ce terme dans le présent article, en gardant à l'esprit les inflexions et résonances locales de ces labellisations sur nos terrains. Sur la sciencefiction en tant que " champ de discours intersectés ", voir Sibela Forrester and Yvonne Howell, «Introduction : From Nauchnaia Fantastika to Post-Soviet Dystopia », Slavic Review, 72 (2), 2013, p. 219-223 ; sur les frontières et la place du « fantastique », voir Edward James, Science Fiction in the Twentieth Century, Oxford : OUP, 1994 ; James E.Gunn \& Matthew Candelaria, eds., Speculations on Speculation: Theories of Science Fiction, Lanham : Scarecrow Press, 2005.

2. Sonija Fritsche, "Introduction », in Id., Science Fiction Literature in East Germany, Bern : Peter Lang, 2006, p. 25.

3. Pour une lecture du genre recadrée dans les partitions de la guerre froide, voir Patrick Major, " Future Perfect? Communist Science Fiction in the Cold War ", in Patrick Major, Rana Mitter, eds., Across the Blocs: Cold War Cultural and Social History, London : Franck Cass, 2004, p. 71-96; David Seed, American Science Fiction and the Cold War: Literature and Film, Edinburgh : EUP, 1999.

4. Eric Hobsbawm, Terence Ranger, eds., The Invention of Tradition, Cambridge : Cambridge University Press, 1983.

5. Blaise Wilfert-Portal, « La place de la littérature étrangère dans le champ littéraire français autour de $1900 »$, Histoire \& mesure, 23 (2), 2008, p. 69-101 ; id., " L'histoire culturelle de l'Europe d'un point de vue transnational», Revue Sciences/Lettres, (1), 2013, http:// rsl.revues.org/279.

6. Edgar Allan Poe, Villiers de l'Isle-Adam, Gustav Meyrink et l'expressionisme littéraire et cinématographique allemand.

7. H. Heinz Ewers.

8. Jules Verne, H.G. Wells.

9. Matthias Schwartz, « How Nauchnaia Fantastika Was Made » ; voir aussi

10. Astronautes, le roman de Stanislaw Lem, paru en 1951, est traduit en bulgare en 1958 et en roumain en 1964. C'est par ailleurs en 1958 que les frères Strugackij font leur apparition dans la revue Science et technique pour la jeunesse en Bulgarie et dans la Collection des récits scientifico-fantastiques en Roumanie.

11. Asif Siddiqi, The Red Rockets' Glare : Spaceflight and the Soviet Imagination, 1957-1967, New York : CUP, 2010 ; Trevo Rockwell, « The Molding of the Rising Generation : Soviet Propaganda and the Hero-Myth of Iurii Gagarin », Past Imperfect, 12, 2006, p. 1-34 ; Slava Gerovitch, " "New Soviet Man" inside Machine: Human Engineering, Spacecraft Design, and the Construction of Communism », Osiris, 22, 2007, p. 134-157; Cathleen Lewis, "The Red Stuff : A History of the 
Public and Material Culture of Early Human Spaceflight in the USSR », PhD. Dissertation, George Washington University, 2008.

12. Donald J. Raleigh, ed., Russia's Sputnik Generation : Soviet Baby Boomers Talk about their Lives, Bloomington : Indiana University Press, 2006.

13. Todor Jalămov, « Fenzinăt "Tantra” », Efremovski sbornik, Sofia : Klub Efremov, 2009.

14. Ibid.

15. Ibid. Si Jurij Gagarin ne se rend pas en Roumanie, Bucarest accueillera en 1961 German Stepanovič Titov, cosmonaute du deuxième vol orbital de l'ère spatiale (Vostok 2, 1961). En 1970, c'est au tour de l'équipage américain d'Apollo-12 de recevoir les honneurs de l'État roumain.

16. Jusqu'à la fin du communisme, les frontières du genre demeureront poreuses.

17. Elle rappelle en cela le succès de la revue bimensuelle Collection des récits scientifico-fantastique en Roumanie entre 1955 et 1974.

18. Les données relatives au tirage ont été obtenues en croisant les informations fournies sur la jaquette des ouvrages et les témoignages d'acteurs de l'époque relatifs aux rééditions.

19. Aux alentours d'un demi-lev en Bulgarie jusqu'au milieu des années 1980 ; en Roumanie, 1 leu entre 1955 et 1974.

20. Plamăk et Septemvrii en Bulgarie ; România literară et Vatra Românească en Roumanie.

21. Dans la seconde moitié des années 1970, une bifurcation s'amorcera toutefois entre les deux pays : là où la populaire Collection de récits scientifico-fantastiques roumaine est supprimée en 1974, officiellement pour cause de pénurie de papier (il faut attendre 1982 pour qu'un supplément annuel de la revue Science et technique soit de nouveau dédié au genre), en Bulgarie le travail de construction éditoriale de la noblesse de la SF se poursuit.

22. Ljuben Dilov a profondément remanié ce texte dans la réédition de 1979 ; le personnage américain y perd sa centralité, l'intrigue étant articulée autour d'un héros bulgare.

23. Crişan Făgeraşu, « O iubire în anul 41042 [Un amour en l'an 41042] », CPSF, 83-84, 1958.

24. Vladimir Colin, « Giovanna et l'ange » (paru en roumain en 1966), in Id., dir., Les meilleures histoires de science-fiction roumaine, Bruxelles : Marabout, 1975, p. 73-104, p. 102.

25. Victor Kernbach, Luntrea sublimă [Le vaisseau sublime], Bucarest : Éditions de la Jeunesse, 1961.

26. Adrian Rogoz " L'Autel des dieux stochastiques ", in Colin, dir., Les meilleures histoires de science-fiction roumaine, p. 153-164, ici p. 156.

27. Adrian Rogoz, « Alambaï ou les arcanes de l'art », in Ibid., p. 175-186, ici p. 174.

28. Pavel Vežinov, Barierata ; Belijat gušter [La barrière ; le lézard blanc], Sofia : Bălgarski pisatel, 1977.

29. Eugen Stancu, « Engineering the Human Soul - Science Fiction in Communist Romania 1955 1989 », Doctoral Thesis in Comparative History of Central, Southeastern and Eastern Europe, Budapest, Central European University, 2011, p. 72.

30. Jenny Andersson, "The Great Future Debate and the Struggle for the World», American Historical Review, déc. 2012, p. 1411-1430.

31. Maliţa aborde lui-même la prospective dans un ouvrage à grande notoriété : Mircea MaliŢa, Cronica anului 2000 [La chronique de l'an 2000], Bucarest : Editura Politică, 1969.

32. Anderson, "The Great Future Debate... », p. 1427.

33. Seuls quelques membres de la nomenklatura (notamment l'Institut des théories sociales contemporaines, ISST, créé en 1968 pour lutter contre les " déviations idéologiques » et placé sous la double tutelle du Comité central et du Présidium de l'Académie des sciences) ont alors accès aux enceintes du dialogue Est-Ouest sur le futur. Les auteurs remercient Liliana Deyanova pour ses remarques sur le rôle de l'ISST.

34. En 1972, Angel Todorov, qui se vante d'avoir rencontré aux États-Unis l'élite de Harvard, Columbia et Princeton, fait paraître une synthèse sur la futurologie occidentale : Angel Todorov, 
Futurologija i ideologija [Futurologie et idéologie], Sofia : Partizdat, 1972 ; Petko Gančev, Futurologija i prognostika [Futurologie et prognostika], Sofia : Nauka i izkustvo, 1973.

35. Mihai Botez, Românii despre ei înşisi [Les Roumains à propos d'eux-mêmes], Bucarest : Litera, 1992.

36. Un laboratoire de recherches prospectives près l'Académie roumaine existait déjà, que dirigeait Mihai Botez. Botez, Românii despre ei înşişi.

37. Darko Suvin, Metamorphoses of Science Fiction: On the Poetics and History of a Literary Genre, New Haven and London : Yale University Press, 1979.

38. Ion Hobana, « Oameni şi stele [Des hommes et des étoiles]», CPSF , 224, 1965.

39. Ljuben Dilov, Pătijat na Ikar [Le chemin d'Icare], Plovdiv : I. Hristo danov, 1974.

40. George Anania, Romulus Bărbulescu, Ferma oamenilor de piatră [La ferme des hommes en pierre], Bucarest : EJ, 1967.

41. Asif Siddiqi, "From Cosmic Enthusiasm to Nostalgia for the Future », in Eva Maurer et al., Soviet Space Culture : Cosmic Enthusiasm in Socialist Societies, New York : Palgrave \& McMillan, 2011, p. 283-306.

42. Un second vol spatial bulgaro-russe aura lieu en 1988 .

43. On notera toutefois la fondation à Sofia dès 1962 d'un éphémère club " Les amis du futur » près le comité sofiote du Komsomol, le lancement en 1969 d'un club-séminaire des jeunes auteurs par la revue Kosmos et la création de groupes de discussion dans plusieurs lycées de Sofia, ainsi qu'à l'université de Sofia à la fin des années 1960.

44. Almanah Anticipaţia, 1989, p. 5. D’autres sources évoquent 4000 à 5000 passionnés autour des clubs : http://jurnalul.ro/scinteia/special/fenomenul-sf-reviste-ilegale-si-zeci-de-cenacluri519106.html. En Bulgarie, en 1988 la revue FEP recense 80 clubs de 3000 membres : FEP, 6, 1988, p. 13-15.

45. Les premiers « cénacles » roumains sont fondés en 1969-1970 à Bucarest (le cénacle SF du Club MM, acronyme qui renvoie à l'an 2000, créé en 1969, rebaptisé Solaris en 1973), à Timişoara (H.G. Wells) et à Craiova (Henri Coandă). Au triangle roumain répondent en Bulgarie les clubs de Sofia (Club Efremov, 1974), Pazardžik (Club Arkadij et Boris Strugackij, 1975), Varna (Andromeda-Observatoire astronomique, 1976) et Plovdiv (Club " XxI siècle », 1977). Sur le cas roumain, voir Stancu, « Engineering the Human Soul...». Sur les cénacles littéraires, Ioana Macrea-Toma, InstituŢii literare în comunismul românesc [Institutions littéraires sous le communisme roumain], Cluj-Napoca : Casa cărŢii de stiinŢă, 2009 ; sur le cas bulgare, voir Efremovski sbornik, Sofia : Klub Efremov, 2009 ; Nepoznatite Strugacki. Jubileen sbornik [Les Strugackij inconnus : mélanges], Pazardžik : Ernor, 2010 et Nikolaj Hristov, « Klub "Dvadeset i părvi vek" [Club xxi siècle], in Agop Melkonjan, dir., Fantastika-1, Sofia : Narodna Mladež, 1985.

46. Todor Jalămov, chimiste de profession et membre du Club Efremov, était ainsi le fils d'un officier de l'aviation formé en URSS et d'une météorologue d'origine russe.

47. L'un des traits singuliers du cas bulgare réside dans le lancement dès 1967 d'un mouvement d'encouragement à la créativité scientifique (Tehničesko i naučno tvorčestvo na mladežta [L'œuvre scientifique et technique de la jeunesse], qui, généreusement doté, maillera peu à peu l'ensemble du territoire. Certains cénacles de science-fiction y seront accueillis.

48. Le Club Efremov organise en 1976 la première rétrospective du cinéma SF grâce à des emprunts auprès d'ambassades, d'instituts culturels étrangers et de la Filmothèque nationale (http://bgf.zavinagi.org/index.php). À Bucarest, le cénacle MM propose en juillet 1969 la projection du documentaire «Apollo 8 " réalisé par la NASA (Colecţia Povestiri ştiinŢ ifico-Fantastice, 354, 15.08. 1969). Des films tels que Shining de Kubrick sont projetés dans les clubs. 
49. Ivajlo Runev, «Bălgarskijat fendăm : dati i săbitija [Les fanzines bulgares : dates et événements]", in : Efremovski sbornik ; Mircea Opriד̧ă, " Cenacluri », 15.03.2010, http:// www.srsff.ro/2010/03/cenacluri-mircea-oprita/ ; Stancu, " Engineering the Human Soul... ».

50. En Bulgarie comme en Roumanie, l'évaluation des manuscrits de SF par des comités éditoriaux constitués de " littéraires » contribue à créer une efficace barrière à l'entrée dans ce champ. La frustration devant les réticences des lettrés à élargir l'accès aux milieux éditoriaux transparaît dans Ivajlo Runev, « 15 let tomu spustya [Quinze ans plus tard] », in 15 let Integraljnji klub fantastiki, evristiki i prognostiki im. Ivana Efremova [Les quinze ans du Club intégral de fantastika, d'heuristique et de prognostika Ivan Efremov], Sofia : GDM, 1989, p. 34-45.

51. Modeli est ainsi édité à 1000 exemplaires (1980), 1200 (1981), 1500 (1989) par le Club Efremov. Si le premier numéro du fanzine roumain Solaris paraît en 90 exemplaires en 1969, l'arrivée des photocopieuses dans les années 1970-80 portera jusqu'à plusieurs milliers le tirage de ces publications. Colecţia " Povestiri ştiinţifico-Fantastice», 354, 15.08.1969 ; Diljan Blagov, " Bibliografija na klubnite izdanija (1977-2009)», Efremovski sbornik.

52. À l'instar de Jordan Radičkov, un temps vice-président de l'Union des écrivains, de Pavel Vežinov ou de Haim Oliver.

53. Le parcours de Stefan Dičev (1920-1996), ingénieur et juriste, auteur de littérature pour enfants est ici éclairant. Un des fondateurs de la prestigieuse collection « Les classiques mondiaux » de Culture populaire, il dirigera la revue Kosmos entre 1962 et 1990.

54. En mai 1989, le Club Efremov possède une bibliothèque de « 600 volumes en bulgare, 1000 en russe, 500 en anglais, français, italien et autres » (selon Ivajlo Runev).

55. Atanas Slavov, Efremovski sbornik, p. 3.

56. Ioana Popa, Traduire sous contraintes : Littérature et communisme (1947-1989), P. : CNRS Éditions, 2010.

57. Dorin Davideanu, « Science fiction britanic în România », à l'adresse :

58. Témoignage de Ion Hobana, http://voyagerblog.ro/2013/02/21/ion-hobana-un-aristocrat-alanticipatiei-romanesti/.

59. Il n'est pas non plus à exclure que la timide internationalisation des écrits bulgares avant 1944 (hors d'Allemagne) explique la faible familiarité des éditeurs et lecteurs étrangers avec la littérature bulgare socialiste, tout comme le déficit de ressources relationnelles des intermédiaires communistes.

60. Krassimir Kavaldjiev, Le marché du livre bulgare en France, mémoire de DESS de relations interculturelles, Paris, université Paris III, 2000.

61. Ses écrits devaient recevoir l'imprimatur des pouvoirs bulgares et être édités par la maison d'édition du parti : Igor Bestužev-Lada, Svetăt prez 2000 godina : Problemi i reşenija [Le monde en l'An 2000 : problème et solutions], Sofia : Partizdat, 1984. Dès 1976, les lecteurs roumains avaient pu se familiariser avec sa définition de la «prognostika» dans le recueil issu de la conférence « futurologique » de Bucarest de 1972 : Igor Bestujev-Lada, « Introducere în analiza sistemică a prognozei sociale considerate ca o categorie [Introduction à l'analyse systémique de la prognose sociale envisagée comme une catégorie]", in Mihai Botez, Mircea Ioanid, dir., Viitorul comun al oamenilor [L'avenir commun des hommes], Bucarest : Editura Politică, 1976, p. 30-50.

62. Almanah Anticipaţia, 1986, p. 81-93.

63. Constantin Cozmiuc, "Timpul este umbra noastră [Le temps est notre ombre] », Almanah Anticipaţia, 1989, p. 165-174.

64. Katherine Verdery, National Ideology under Socialism: Identity and Cultural Politics in Ceauşescu's Romania, Berkeley : University of California Press, 1991 ; sur le cas bulgare, voir Tchavdar Marinov, La Thrace ancienne dans l'imaginaire moderne : Aspects idéologiques de la construction des études thraces en Europe du Sud-Est (Roumanie, Grèce, Bulgarie), mémoire de $3^{\mathrm{e}}$ année présenté à l'Académie des Inscriptions et Belles-Lettres, Athènes : EFA, 2013, 145 p. 
65. Non sans susciter, à l'occasion, des relectures satiriques des rêves de grandeur dans la Roumanie des années 1980. Voir Alexandru Ungureanu, « Renaşterea poetului [La Renaissance du poète] ", Almanah AnticipaŢia, 1989, p. 39-42, p. 41.

66. Sur ces nouvelles articulations entre passé, présent et futur, voir Nadège Ragaru, « Au-delà des étoiles : Stars Wars et l'histoire culturelle du socialisme tardif en Bulgarie ", Cahiers du Monde russe, 54 (1-2), 2013, p. 353-382 (esp. p. 368-373).

67. CFSP, 444, 15.05.1973.

68. FEP, 6, 1988, p. 8-11.

69. Alexandru Ungureanu, «Blestemul [La malédiction] » et " Moartea lui LişiŢă [La mort de LişiŢă] », Almanah AnticipaŢia, 1989, p. 37-47.

70. Todor Nenov, « Nošt e... [Il fait nuit...] », FEP, 6, 1988, p. 4-6 et 11.

71. FEP, 3, 1988, p. 4.

72. Rosica Panajotova, « Anketa na spisanije "FEP" [Enquête du magazine FEP] », FEP, 6, 1988, p. 22-23.

73. Bernice Glatzer-Rosenthal, ed., The Occult in Russian and Soviet Culture, Ithaca, N.Y. : CUP, 1997 ; Birgit Menzel et al., eds., The New Age of Russia: Occult and Esoteric Dimensions, Munich : Verlag Otto Sagner, 2012.

74. Mircea Eliade, Yoga, essai sur les origines de la mystique indienne, Bucarest - Paris : P. Geuthner, 1936 ; id, Techniques du Yoga, P. : Gallimard, 1948. Sur la complexe trajectoire biographique d'Eliade, voir Florin Ţurcanu, Mircea Eliade, Le prisonnier de l'histoire, P. : La Découverte, 2003.

75. Birgit Menzel, " The Occult Revival in Russia Today and its Impact on Literature ", The Harriman Review, 16 (1), 2007, p. 3.

76. Matthias Schwartz, Die Erfindung des Kosmos: Zur sowjetischen Science Fiction and populärwissenschaftlichen Publizistik vom Sputinikflug vis zum Ende der Tauwetterzeit, Frankfurt : Petar Lang, 2003.

77. Voir les recherches menées par la Rand aux États-Unis : P.T. Van Dyke and M.L.Juncosa, "Paranormal Phenomena. Briefing on a Net Assessment Study», Santa Monica: Rand, 1973 à l'adresse :

78. Robert C. Williams, "Mysticism and Money: Nicholas Roerich», in id., Russian Art and American Money, 1900-1940, Cambridge : HUP, 1980, p. 11-46.

79. Mihail Gruev, « Ljudmila Živkova, pătijat kăm “Agni Joga” [Ljudmila Živkova, le chemin vers “Agni Joga"] », in Prelomni vremena. Jubileen sbornik v čest na 65-godišninata na prof. Ljubomir Ognjanov [Des temps de rupture : mélanges en l'honneur du professeur Liubomir Ognjanov pour son soixante-cinquième anniversaire], Sofia : UI 'Kliment Ohridski', 2006, p. 796-816.

80. Ivan Elenkov, Kulturnijat front [Le front de la culture], Sofia : Siela, 2008, p. 317-337.

81. Mircea Eliade, « Nopţi la Serampore (fragment din volumul Secretul doctorului Honigberger) [Des Nuits à Serampore (fragment du volume Le secret du docteur Honigberger] », in Ion Hobana, Vârsta de aur anticipaţiei românesti [L'Age d'or de l'anticipation roumaine], Bucarest : Ed. Tineretului, 1969 ; Elaine L. Kleiner, "Romanian "Science Fantasy" in the Cold War Era ", Science Fiction Studies, 19 (1) 1992, p. 59-68.

82. Non sans difficultés, comme en témoignent les écarts de parution des trois volumes : Mircea Eliade, Istoria credinţelor şi ideilor religioase [L'Histoire des croyances et des idées religieuses], Bucarest : Editura ştiinţifică şi Enciclopedică, 3 vol. , 1981, 1986 et 1988.

83. Sur le cas soviétique, voir Victoria Smolkin-Rothrock, "The Ticket to the Soviet Soul: Science, Religion, and the Spiritual Crisis of Late Soviet Atheism », The Russian Review, 73, avril 2014, p. 171-197.

84. Hristo Gešanov, « Covekăt na bădešteto se izgražda dnes [L'homme du futur se construit aujourd'hui] », in : Efremovski sbornik.

85. Entretien avec A. Slavov, Sofia, 01.03.2012. 
86. FEP, 6, 1988, p. 15.

87. Voir notamment le succès populaire du livre du physicien Nicolae Tufoi, Yoga, Izvor de sănă tate [Le yoga, une source de santé], publié par la respectable maison d'édition Junimea en 1978.

88. Doina Jela et al., Afacerea Medita Ţia Transcendentală [L'Affaire "la médition transcendentale"], Bucarest : Humanitas, 2004.

89. Galia Valtchinova, « Between Ordinary Pain and Extraordinary Knowledge: The Seer Vanga in the Everyday Life of Bulgarians during Socialism (1960s-1970s) », Aspasia, 3, 2008, p. 106-130.

90. Communication par courriel de l'historien Mihail Gruev, 20.11.2014.

91. Gabriel Andreescu, Reprimarea Mişcării Yoga în Anii '80 [La répression contre le mouvement yoga dans les années 1980], Iaşi : Polirom, 2008.

92. Alexandru Mironov, «O aventură numită Anticipa ţia [Une aventure appeléee Anticipation] », Almanah Anticipaţia, 2013, p. 2-3.

93. Kosmos, 1987, 2, p. 18-19.

94. Val Todorov, "V Klub namerih sebe si [Dans le club, je me suis trouvé ]», Efremovski sbornik.

95. DănuŢ Ungureanu, « Cum m-am facut Solarisian [Comment j'ai rejoint le club Solaris]», à l'adresse : http://www.srsff.ro/2013/04/cum-m-am-facut-solarisian-episodul-2/

96. Davideanu, Science fiction britanic în România.

97. Ungureanu, « Cum m-am facut Solarisian ».

98. Davideanu, Science fiction britanic în România.

99. FEP, 1, 1989, p. 30-31.

100. FEP, 6, 1988, p. 12.

101. FEP, 6, 1988, p. 3.

102. Miglena Nikolchina, "The West as Intellectual Utopia», in Maria Todorova, ed., Remembering Communism: Genres of Representation, New York : Social Science Research Council, 2010, p. 95-126. (esp. p. 113, 115).

103. FEP, 6,1988 , p. 22-25.

104. Reinhart Koselleck, Le Futur passé : Contribution à la sémantique des temps historiques, P. : EHESS, 1990.

\section{RÉSUMÉS}

L'article propose une réflexion sur les temporalités socialistes en Bulgarie et en Roumanie à travers le prisme de la science-fiction, un genre habité de visions et de prodiges, qui fut aussi un support privilégié d'interrogation sur les présents socialistes. Étudiée à travers ses producteurs, ses circulations, ses lecteurs et ses clubs d'amateurs, la science-fiction permet en effet de retracer les croisements entre productions politiques, thématisations littéraires et expériences sociales du temps. Ce faisant, l'article suggère la faible pertinence de découpages Est-Ouest pour appréhender l'histoire des sensibilités, de la science ou les interrogations sur le devenir de la planète. Il montre toutefois aussi que les traversées Est-Ouest des textes et des savoirs empruntèrent d'un pays à l'autre des sentiers différents, tributaires des histoires ante communistes et de visions nationales contrastées de la direction spatio-temporelle de la modernité. Au-delà de ces divergences, les univers SF suggèrent des chronologies proches du (dés)enchantement socialiste : à une projection plutôt confiante dans les années 1960 succéda au 
cours des années 1980 un éclatement générationnel, social et territorial des visions du présent et de l'avenir. Le repli vers un lointain passé nationalisé ou un futur inaccessible n'empêcha cependant pas les clubs SF de fournir, particulièrement en Bulgarie, un espace de débats sur un futur socialiste réformé.

This article explores the socialist experiences of time in Bulgaria and Romania through the prism of literary science fiction, a genre inhabited with visions and wonders, which was also a site of discussion about the socialist presents. Envisioned through its producers, circulations, readership and amateur clubs, science fiction offers a vantage point on the interplay between political productions, literary thematizations and social experiences of time. The article also suggests the low relevance of East-West divisions in reconstructing the history of sensibilities and science or the debates over the planet's future. The paper, however, shows that trans-bloc circulations of texts and knowledge followed different paths in Bulgaria and Romania; these routes were indeed shaped by the two countries' respective ante-communist histories and different visions of the spatio-temporal direction of modernity. Beyond these contrasts, a survey of science fiction writings and publics suggests similar chronologies of socialist (dis)enchantment in both countries: to a rather confident projection into the future in the 1960s succeeded a generational, social and territorial diversification of visions of the present and future. However, the retreat to a distant nationalized past or an inaccessible future did not prevent science fiction clubs from providing, particularly in Bulgaria, a space for debate on a possibly reformed socialist future.

\section{AUTEURS}

\section{NADĖGE RAGARU}

Sciences Po-CERI, nadege.ragaru@sciencespo.fr

\section{ANTONELA CAPELLE-POGĂCEAN}

Sciences Po-CERI, antonela.capellepogacean@sciencespo.fr 